\title{
CRYSTAL BASES FOR QUANTUM AFFINE ALGEBRAS AND COMBINATORICS OF YOUNG WALLS
}

\author{
SEOK-JIN KANG*
}

\begin{abstract}
In this paper, we give a realization of crystal bases for quantum affine algebras using some new combinatorial objects which we call the Young walls. The Young walls consist of colored blocks with various shapes that are built on the given ground-state wall and can be viewed as generalizations of Young diagrams. The rules for building Young walls and the action of Kashiwara operators are given explicitly in terms of combinatorics of Young walls. The crystal graphs for basic representations are characterized as the set of all reduced proper Young walls. The characters of basic representations can be computed easily by counting the number of colored blocks that have been added to the ground-state wall.
\end{abstract}

\section{INTRODUCTION}

The crystal basis theory for integrable modules over quantum groups was introduced by Kashiwara using a combinatorial method ([10, 11]). In [16], a more geometric approach was developed by Lusztig and is called the canonical basis theory. The crystal bases can be viewed as bases at $q=0$ and they are given a structure of colored oriented graph, called the crystal graphs, with arrows defined by the Kashiwara operators. The crystal graphs have many nice combinatorial features reflecting the internal structure of integrable modules over quantum groups. For instance, one of the major goals in representation theory is to find an explicit expression for the characters of representations and this goal can be achieved by finding an explicit combinatorial description of crystal bases. Moreover, the crystal bases have extremely nice behavior with respect to taking the tensor product. The action of the Kashiwara operators is given by the tensor product rule and the irreducible decomposition of the tensor product of integrable modules is equivalent to decomposing the tensor product of crystal graphs into a disjoint union of connected components. Thus the crystal basis theory provides us with a very powerful combinatorial method of studying the structure of integrable modules over quantum groups.

*This research was supported by KOSEF Grant \# 98-0701-01-5-L and the Young Scientist Award, Korean Academy of Science and Technology. 
In [17], Misra and Miwa constructed the crystal bases for basic representations of quantum affine algebras $U_{q}\left(A_{n}^{(1)}\right)$ using the Fock space representation on the space of Young diagrams with colored boxes. The crystal graphs were characterized as the set of n-reduced Young diagrams (with colored boxes). Their idea was extended to construct crystal bases for irreducible highest weight $U_{q}\left(A_{n}^{(1)}\right)$-modules with arbitrary higher level [6]. The crystal graphs constructed in [6] and [17] can be parametrized by certain paths which arise naturally in the theory of solvable lattice models.

Motivated by this observation, Kang, Kashiwara, Misra, Miwa, Nakashima and Nakayashiki developed the theory of perfect crystals for general quantum affine algebras and gave a realization of crystal graphs for irreducible highest weight modules over classical quantum affine algebras with arbitrary higher levels in terms of paths $([8,9])$. In this way, the theory of vertex models can be explained in the language of representation theory of quantum affine algebras and the 1-point function of the vertex model was expressed as the quotient of the string function and the character of the corresponding irreducible highest weight representation.

On the other hand, in [15], Lascoux, Leclerc and Thibon discovered a surprising connection between the crystal basis theory for quantum affine algebras and modular representation theory of Hecke algebras. For a complex number $\zeta$, let $H_{N}(\zeta)$ denote the Hecke algebra of type $A_{N-1}$. It is known that the indecomposable modules over $H_{N}(\zeta)$, called the Specht modules, are parametrized by Young diagrams with $N$ boxes. We denote by $S(Y)$ the Specht module corresponding to the Young diagram $Y$ with $N$ boxes. If $\zeta$ is not a root of unity, then the category of finite dimensional $H_{N}(\zeta)$-modules is semisimple, and hence the set of Specht modules coincides with the set of irreducible modules. However, if $\zeta$ is a primitive $n$-th root of unity, then the Specht modules are no longer irreducible, and the irreducible $H_{N}(\zeta)$-modules are characterized as the unique iireducible quotients of the Specht modules corresponding to the $n$-reduced Young diagrams with $N$ boxes (see, for example, [2, 3]).

Recall that an $n$-reduced Young diagram $Y$ with $N$ boxes can be viewed as a crystal basis element of a basic representation of the quantum affine algebra $U_{q}\left(A_{n}^{(1)}\right)$. Using the $U_{q}\left(A_{n}^{(1)}\right)$-module action on the space of all Young diagrams (see, for example, [6, 17]), one should be able to write the global basis element (or canonical basis element) $G(Y)$ as a linear combination of Young diagrams:

$$
G(Y)=\sum_{Y^{\prime}} d_{Y^{\prime}, Y}(q) Y^{\prime} \quad \text { for some } \quad d_{Y^{\prime}, Y}(q) \in \mathbf{C}[q]
$$

In [15], Lascoux, Leclerc and Thibon gave a recursive algorithm of computing the polynomials $d_{Y^{\prime}, Y}(q)$ and conjectured that 


$$
d_{Y^{\prime}, Y}(1)=\left[S\left(Y^{\prime}\right): D(Y)\right]
$$

where $D(Y)$ is the irreducible $H_{N}(\zeta)$-module corresponding to an $n$-reduced Young diagram $Y, S\left(Y^{\prime}\right)$ is the Specht module corresponding to a Young diagram $Y^{\prime}$, and $\left[S\left(Y^{\prime}\right): D(Y)\right.$ ] denotes the multiplicity of $D(Y)$ in a composition series of $S\left(Y^{\prime}\right)$. This conjecture was proved by Ariki ([1]). In [四, Goodman and Wenzl found a faster algorithm of computing the polynomials $d_{Y^{\prime}, Y}(q)$ and gave a combinatorial proof of the fact that the polynomials $d_{Y^{\prime}, Y}(q)$ coincide with the affine Kazhdan-Lusztig polynomials.

The purpose of this paper is to give a realization of crystal bases for quantum affine algebras using some new combinatorial objects which we call the Young walls. The Young walls consist of colored blocks with various shapes that are built on the given ground-state wall and can be viewed as generalizations of Young diagrams. For the classical quantum affine algebras of type $A_{n}^{(1)}(n \geq 1)$, $A_{2 n-1}^{(2)}(n \geq 3), D_{n}^{(1)}(n \geq 4), A_{2 n}^{(2)}(n \geq 2), D_{n+1}^{(2)}(n \geq 2)$ and $B_{n}^{(1)}(n \geq 3)$, the rules for building Young walls and the action of Kashiwara operators are given explicitly in terms of combinatorics of Young walls. They are quite similar to playing with LEGO blocks and the Tetris game. The crystal graphs for basic representations are characterized as the set of all reduced proper Young walls. The characters of basic representations can be computed easily by counting the number of colored blocks in reduced proper Young walls that have been added to the ground-state wall.

We expect that we will be able to define the $U_{q}(\mathfrak{g})$-module action on the space of all proper Young walls and find an effective algorithm for computing the global basis element corresponding to each reduced proper Young wall. For some mysterious reason, it is more difficult to deal with the quantum affine algebras of type $C_{n}^{(1)}$ than the other classical quantum affine algebras. In [5], we gave a characterization of crystal graphs for basic representations of $U_{q}\left(C_{2}^{(1)}\right)$ as the set of reduced proper Young walls. But it still remains to extend the results of [5] to quantum affine algebras $U_{q}\left(C_{n}^{(1)}\right)$ for $n \geq 3$. As the representation theory of Hecke algebras can be explained in terms of Young diagrams, we also expect that there exists an interesting algebraic structure related to the combinatorics of Young walls such that the irreducible modules at some specialization are parametrized by reduced proper Young walls and that the decomposition matrices are determined by the polynomials giving the global basis elements.

This paper is organized as follows. In Section 1, we fix the notations and recall the basic definitions on quantum affine algebras. In Section 2, we review some of the fundamental properties of crystal bases for integrable modules over quantum groups. The tensor product rule for Kashiwara operators is 
explained using the $i$-signatures. The definition of perfect crystals is given in Section 3. We also include the examples of perfect crystals of level 1. In Section 4, we recall one of the main results of [8] and [9]: the path realization of crystal graphs. The crystal structure on the set of paths is given by the tensor product rule. We also demonstrate the top part of crystal graphs for basic representations of quantum affine algebras of some small ranks.

Section 5 and Section 6 are devoted to the combinatorics of Young walls. In Section 5, we define the notion of ground-state walls, Young walls and proper Yong walls, and give an explicit description of the rules and patterns for building Young walls. In Section 6, we define an affine crystal structure on the set of all proper Young walls. We also show that the set of all reduced proper Young walls is stable under the action of Kashiwara operators.

Finally, in Section 7, we prove the main result of this paper (Theorem 7.1). We prove that the crystal graph $B(\lambda)$ of the basic representation is isomorphic to the affine crystal $\mathbf{Y}(\lambda)$ consisting of reduced proper Young walls built on the ground-state wall $Y_{\lambda}$. The path realization of crystal graphs plays a crucial role in the proof of our theorem. At the end of Section 7, we give some examples of the affine crystal $\mathbf{Y}(\lambda)$ consisting of reduced proper Young walls.

Acknowledgments. The author would like to thank Jin Hong for many valuable discussions and his generous help in making the diagrams in this paper. Part of this work was completed while the author was visiting Massachusetts Institute of Technology, Yale University and Korea Institute for Advanced Study in the year of 1999. He is very grateful to all the faculty and staff members of these institutions for their hospitality and support during his visit.

\section{Quantum AFFine Algebras}

Let $I=\{0,1, \cdots, n\}$ be an index set and let $A=\left(a_{i j}\right)_{i, j \in I}$ be a generalized Cartan matrix of affine type. Consider a free abelian group of rank $n+2$

$$
P^{\vee}=\mathbf{Z} h_{0} \oplus \mathbf{Z} h_{1} \oplus \cdots \oplus \mathbf{Z} h_{n} \oplus \mathbf{Z} d
$$

and let $\mathfrak{h}=\mathbf{C} \otimes_{\mathbf{z}} P^{\vee}$ be its complexification. The free abelian group $P^{\vee}$ is called the dual weight lattice and the complex vector space $\mathfrak{h}$ is called the Cartan subalgebra.

We define the linear functionals $\alpha_{i}$ and $\Lambda_{i}(i \in I)$ on $\mathfrak{h}$ by

$$
\begin{array}{ll}
\alpha_{i}\left(h_{j}\right)=a_{j i}, & \alpha_{i}(d)=\delta_{0, i}, \\
\Lambda_{i}\left(h_{j}\right)=\delta_{i j}, & \Lambda_{i}(d)=0 \quad(i, j \in I) .
\end{array}
$$


The $\alpha_{i}$ (resp. $h_{i}$ ) are called the simple roots (resp. simple coroots) and the $\Lambda_{i}$ are called the fundamental weights. We denote by $\Pi=\left\{\alpha_{i} \mid i \in I\right\}$ (resp. $\Pi^{\vee}=\left\{h_{i} \mid i \in I\right\}$ ) the set of simple roots (resp. simple coroots). The affine weight lattice is defined to be

$$
P=\left\{\lambda \in \mathfrak{h}^{*} \mid \lambda\left(P^{\vee}\right) \subset \mathbf{Z}\right\} .
$$

The quintuple $\left(A, \Pi, \Pi^{\vee}, P, P^{\vee}\right)$ is called an affine Cartan datum. To each affine Cartan datum, we can associate an infinite dimensional Lie algebra $\mathfrak{g}$ called the affine Kac-Moody algebra ([7]). The center of the affine Kac-Moody algebra $\mathfrak{g}$ is 1-dimensional and is generated by the canonical central element

$$
c=c_{0} h_{0}+c_{1} h_{1}+\cdots+c_{n} h_{n} .
$$

Moreover, the imaginary roots of $\mathfrak{g}$ are nonzero integral multiples of the null root

$$
\delta=d_{0} \alpha_{0}+d_{1} \alpha_{1}+\cdots+d_{n} \alpha_{n} .
$$

Here, $c_{i}$ and $d_{i}(i \in I)$ are the non-negative integers given in [7]. Using the fundamental weights and the null root, the affine weight lattice can be written as

$$
P=\mathbf{Z} \Lambda_{0} \oplus \mathbf{Z} \Lambda_{1} \oplus \cdots \oplus \mathbf{Z} \Lambda_{n} \oplus \mathbf{Z} \delta
$$

Set

$$
P^{+}=\left\{\lambda \in P \mid \lambda\left(h_{i}\right) \in \mathbf{Z}_{\geq 0} \quad \text { for all } i \in I\right\} .
$$

The elements of $P$ (resp. $P^{+}$) are called the affine weights (resp. affine dominant integral weights). The level of an affine dominant integral weight $\lambda \in P^{+}$ is defined to be the nonnegative integer $\lambda(c)$.

Example 1.1. In this example, we present the affine Dynkin diagrams, the canonical central elements, the null roots and the level 1 dominant integral weights for affine Cartan data of classical type.

(a) $A_{n}^{(1)}(n \geq 1)$ :

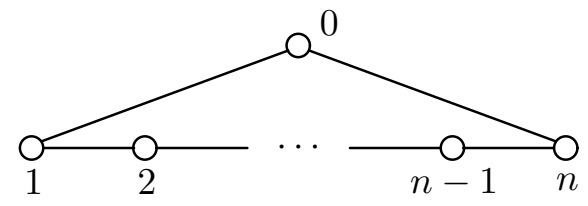

$$
\begin{aligned}
& c=h_{0}+h_{1}+\cdots+h_{n}, \\
& \delta=\alpha_{0}+\alpha_{1}+\cdots+\alpha_{n}, \\
& \lambda=\Lambda_{i} \quad(i \in I) .
\end{aligned}
$$


(b) $A_{2 n-1}^{(2)}(n \geq 3)$ :

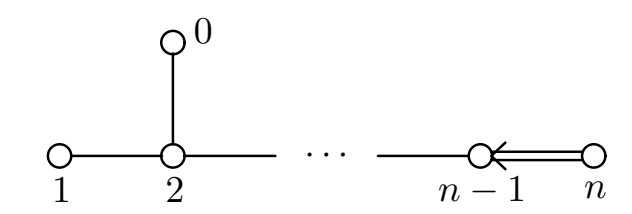

$$
\begin{aligned}
& c=h_{0}+h_{1}+2 h_{2}+\cdots+2 h_{n-1}+2 h_{n}, \\
& \delta=\alpha_{0}+\alpha_{1}+2 \alpha_{2}+\cdots+2 \alpha_{n-1}+\alpha_{n}, \\
& \lambda=\Lambda_{0}, \Lambda_{1} .
\end{aligned}
$$

(c) $D_{n}^{(1)}(n \geq 4)$

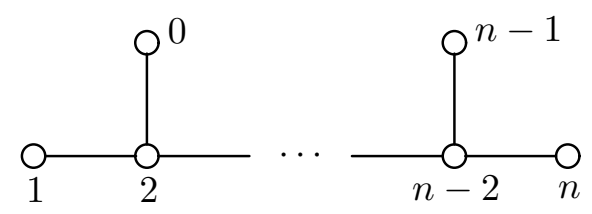

$$
\begin{aligned}
& c=h_{0}+h_{1}+2 h_{2}+\cdots+2 h_{n-2}+h_{n-1}+h_{n}, \\
& \delta=\alpha_{0}+\alpha_{1}+2 \alpha_{2}+\cdots+2 \alpha_{n-2}+\alpha_{n-1}+\alpha_{n}, \\
& \lambda=\Lambda_{0}, \Lambda_{1}, \Lambda_{n-1}, \Lambda_{n}
\end{aligned}
$$

(d) $A_{2 n}^{(2)}(n \geq 2)$

$$
\begin{aligned}
& \underset{0}{\rightleftharpoons}=\mathrm{O}_{2}-\cdots+\underset{n-1}{\mathrm{O}} \\
& c=h_{0}+2 h_{1}+\cdots+2 h_{n-1}+2 h_{n}, \\
& \delta=2 \alpha_{0}+2 \alpha_{1}+\cdots+2 \alpha_{n-1}+\alpha_{n}, \\
& \lambda=\Lambda_{0} \text {. }
\end{aligned}
$$

(e) $D_{n+1}^{(2)}(n \geq 2)$

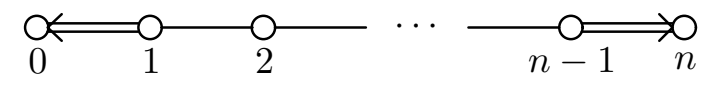




$$
\begin{aligned}
& c=h_{0}+2 h_{1}+\cdots+2 h_{n-1}+h_{n}, \\
& \delta=\alpha_{0}+\alpha_{1}+\cdots+\alpha_{n-1}+\alpha_{n}, \\
& \lambda=\Lambda_{0}, \Lambda_{n} .
\end{aligned}
$$

(f) $B_{n}^{(1)}(n \geq 3)$

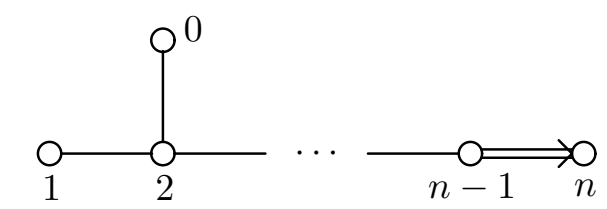

$$
\begin{aligned}
& c=h_{0}+h_{1}+2 h_{2}+\cdots+2 h_{n-1}+h_{n}, \\
& \delta=\alpha_{0}+\alpha_{1}+2 \alpha_{2}+\cdots+2 \alpha_{n-1}+2 \alpha_{n}, \\
& \lambda=\Lambda_{0}, \Lambda_{1}, \Lambda_{n} .
\end{aligned}
$$

We denote by $q^{h}\left(h \in P^{\vee}\right)$ the basis elements of the group algebra $\mathbf{C}(q)\left[P^{\vee}\right]$ with the multiplication $q^{h} q^{h^{\prime}}=q^{h+h^{\prime}}\left(h, h^{\prime} \in P^{\vee}\right)$. Let $(\mid)$ be a nondegenerate symmetric bilinear form on $\mathfrak{h}^{*}$ satisfying

$$
\frac{2\left(\alpha_{i} \mid \alpha_{j}\right)}{\left(\alpha_{i} \mid \alpha_{i}\right)}=a_{i j} \quad \text { for all } \quad i, j \in I
$$

Set $q_{i}=q^{\frac{\left(\alpha_{i} \mid \alpha_{i}\right)}{2}}, \quad K_{i}=q^{\frac{\left(\alpha_{i} \mid \alpha_{i}\right)}{2} h_{i}}$ and define

$$
[k]_{i}=\frac{q_{i}^{k}-q_{i}^{-k}}{q_{i}-q_{i}^{-1}}, \quad[n]_{i} !=\prod_{k=1}^{n}[k]_{i}, \quad\left[\begin{array}{c}
m \\
n
\end{array}\right]_{i}=\frac{[m]_{i} !}{[n]_{i} ![m-n]_{i} !} .
$$

We will also use the following notations:

$$
e_{i}^{(n)}=e_{i}^{n} /[n]_{i} !, \quad f_{i}^{(n)}=f_{i}^{n} /[n]_{i} !
$$

Definition 1.2. The quantum affine algebra $U_{q}(\mathfrak{g})$ associated with the affine Cartan datum $\left(A, \Pi, \Pi^{\vee}, P, P^{\vee}\right)$ is the associative algebra with 1 over $\mathbf{C}(q)$ generated by the symbols $e_{i}, f_{i}(i \in I)$ and $q^{h}\left(h \in P^{\vee}\right)$ subject to the following defining relations: 


$$
\begin{aligned}
& q^{0}=1, \quad q^{h} q^{h^{\prime}}=q^{h+h^{\prime}} \quad\left(h, h^{\prime} \in P^{\vee}\right), \\
& q^{h} e_{i} q^{-h}=q^{\alpha_{i}(h)} e_{i}, \quad q^{h} f_{i} q^{-h}=q^{-\alpha_{i}(h)} f_{i} \quad\left(h \in P^{\vee}, i \in I\right), \\
& e_{i} f_{j}-f_{j} e_{i}=\delta_{i j} \frac{K_{i}-K_{i}^{-1}}{q_{i}-q_{i}^{-1}} \quad(i, j \in I), \\
& \sum_{k=0}^{1-a_{i j}}(-1)^{k}\left[\begin{array}{c}
1-a_{i j} \\
k
\end{array}\right]_{i} e_{i}^{1-a_{i j}-k} e_{j} e_{i}^{k}=0 \quad(i \neq j), \\
& \sum_{k=0}^{1-a_{i j}}(-1)^{k}\left[\begin{array}{c}
1-a_{i j} \\
k
\end{array}\right]_{i} f_{i}^{1-a_{i j}-k} f_{j} f_{i}^{k}=0 \quad(i \neq j) .
\end{aligned}
$$

The subalgebra of $U_{q}(\mathfrak{g})$ generated by $e_{i}, f_{i}, K_{i}^{ \pm 1}(i \in I)$ is denoted by $U_{q}^{\prime}(\mathfrak{g})$, and is also called the quantum affine algebra. Let

$$
\bar{P}^{\vee}=\mathbf{Z} h_{0} \oplus \mathbf{Z} h_{1} \oplus \cdots \oplus \mathbf{Z} h_{n} \quad \text { and } \quad \overline{\mathfrak{h}}=\mathbf{C} \otimes \mathbf{z} \bar{P}^{\vee} .
$$

Consider $\alpha_{i}$ and $\Lambda_{i}(i \in I)$ as linear functionals on $\overline{\mathfrak{h}}$ and set

$$
\bar{P}=\mathbf{Z} \Lambda_{0} \oplus \mathbf{Z} \Lambda_{1} \oplus \cdots \oplus \mathbf{Z} \Lambda_{n} .
$$

For example, $\delta=0$ as an element in $\bar{P}$. The elements of $\bar{P}$ are called the classical weights. The algebra $U_{q}^{\prime}(\mathfrak{g})$ can be regarded as the quantum affine algebra associated with the classical Cartan datum $\left(A, \Pi, \Pi^{\vee}, \bar{P}, \bar{P}^{\vee}\right)$.

The projection $\mathrm{cl}: P \longrightarrow \bar{P}$ will be denoted by $\lambda \longmapsto \bar{\lambda}$ and we will fix an embedding aff $: \bar{P} \longrightarrow P$ such that

$$
\mathrm{cl} \circ \mathrm{aff}=\mathrm{id}, \quad \text { aff } \circ \mathrm{cl}\left(\alpha_{i}\right)=\alpha_{i} \quad \text { for } \quad i \neq 0 .
$$

We define

$$
\bar{P}^{+}=\operatorname{cl}\left(P^{+}\right)=\left\{\lambda \in \bar{P} \mid \lambda\left(h_{i}\right) \geq 0 \text { for all } i \in I\right\} .
$$

The elements of $\bar{P}^{+}$are called the classical dominant integral weights. For simplicity, we will omit the notations for the projection and the embedding. We will state explicitly whether a linear functional is an affine weight or a classical weight whenever it could cause a confusion. A classical dominant integral weight $\lambda \in \bar{P}^{+}$is said to have level $l \in \mathbf{Z}_{\geq 0}$ if $\lambda(c)=l$. Note that it has the same level as its affine counterpart.

\section{CRystal Bases}

In this section, we briefly review the crystal basis theory for quantum affine algebras. The category $\mathcal{O}_{\text {int }}$ consists of $U_{q}(\mathfrak{g})$-modules (resp. $U_{q}^{\prime}(\mathfrak{g})$-modules $M)$ satisfying the properties: 
(i) $M=\bigoplus_{\lambda \in P} M_{\lambda}$ (resp. $M=\bigoplus_{\lambda \in \bar{P}} M_{\lambda}$ ), where

$$
M_{\lambda}=\left\{v \in M \mid q^{h} v=q^{\lambda(h)} v \text { for all } h \in P^{\vee}\left(\text { resp. } h \in \bar{P}^{\vee}\right)\right\},
$$

(ii) for each $i \in I, M$ is a direct sum of finite dimensional irreducible $U_{i^{-}}$ modules, where $U_{i}$ denotes the subalgebra generated by $e_{i}, f_{i}, K_{i}^{ \pm 1}$ which is isomorphic to $U_{q}\left(\mathfrak{s l}_{2}\right)$.

It is known that every $U_{q}(\mathfrak{g})$-module (resp. $U_{q}^{\prime}(\mathfrak{g})$-module) in the category $\mathcal{O}_{\text {int }}$ is a direct sum of irreducible highest weight modules $V(\lambda)$ with $\lambda \in P^{+}$(resp. $\left.\lambda \in \bar{P}^{+}\right)$.

Fix an index $i \in I$. By the representation theory of $U_{q}\left(\mathfrak{s l}_{2}\right)$, every element $v \in M_{\lambda}$ can be written uniquely as

$$
v=\sum_{k \geq 0} f_{i}^{(k)} v_{k}
$$

where $k \geq-\lambda\left(h_{i}\right)$ and $v_{k} \in$ ker $e_{i} \cap M_{\lambda+k \alpha_{i}}$. We define the endomorphisms $\tilde{e}_{i}$ and $\tilde{f}_{i}$ on $M$, called the Kashiwara operators, by

$$
\tilde{e}_{i} v=\sum_{k \geq 1} f_{i}^{(k-1)} v_{k}, \quad \tilde{f}_{i} v=\sum_{k \geq 0} f_{i}^{(k+1)} v_{k} .
$$

It is easy to see that

$$
\tilde{e}_{i} M_{\lambda} \subset M_{\lambda+\alpha_{i}}, \quad \tilde{f}_{i} M_{\lambda} \subset M_{\lambda-\alpha_{i}} \text { for all } i \in I .
$$

Let $M$ be a $U_{q}(\mathfrak{g})$-module (or $U_{q}^{\prime}(\mathfrak{g})$-module) in the category $\mathcal{O}_{\text {int }}$ and let

$$
\mathbf{A}=\{f / g \in \mathbf{C}(q) \mid f, g \in \mathbf{C}[q], g(0) \neq 0\}
$$

be the subring of $\mathbf{C}(q)$ consisting of the rational functions in $q$ that are regular at $q=0$. We now define the notion of crystal bases which was introduced in [10, 11].

Definition 2.1. A free A-submodule $L$ of $M$ is called a crystal lattice if

(i) $L$ generates $M$ as a $\mathbf{C}(q)$-vector space; i.e., $M \cong \mathbf{C}(q) \otimes_{\mathbf{A}} L$,

(ii) $L=\bigoplus_{\lambda \in P} L_{\lambda}\left(\right.$ or $L=\bigoplus_{\lambda \in \bar{P}} L_{\lambda}$ ), where $L_{\lambda}=L \cap M_{\lambda}$,

(iii) $\tilde{e}_{i} L \subset L, \tilde{f}_{i} L \subset L$ for all $i \in I$.

Definition 2.2. A crystal basis of $M$ is a pair $(L, B)$ such that

(i) $L$ is a crystal lattice of $M$,

(ii) $B$ is a $\mathbf{C}$-basis of $L / q L$,

(iii) $B=\bigsqcup_{\lambda \in P} B_{\lambda}$, where $B_{\lambda}=B \cap\left(L_{\lambda} / q L_{\lambda}\right)$,

(iv) $\tilde{e}_{i} B \subset B \cup\{0\}, \tilde{f}_{i} B \subset B \cup\{0\}$,

(v) for $b, b^{\prime} \in B, \tilde{f}_{i} b=b^{\prime}$ if and only if $b=\tilde{e}_{i} b^{\prime}$. 
The set $B$ is given a colored oriented graph structure with the arrow defined by

$$
b \stackrel{i}{\longrightarrow} b^{\prime} \quad \text { if and only if } \quad \tilde{f}_{i} b=b^{\prime} .
$$

The graph $B$ is called the crystal graph of $M$ and it reflects the combinatorial structure of $M$. For instance, we have

$$
\operatorname{dim}_{\mathbf{C}(q)} M_{\lambda}=\# B_{\lambda} \quad \text { for all } \quad \lambda \in P \quad(\text { or } \quad \lambda \in \bar{P}) .
$$

We recall the basic properties of crystal graphs. Let $B$ be a crystal graph for a $U_{q}(\mathfrak{g})$-module (or a $U_{q}^{\prime}(\mathfrak{g})$-module) $M$ in the category $\mathcal{O}_{\text {int }}$. For each $b \in B$ and $i \in I$, we define

$$
\varepsilon_{i}(b)=\max \left\{k \geq 0 \mid \tilde{e}_{i}^{k} b \in B\right\}, \quad \varphi_{i}(b)=\max \left\{k \geq 0 \mid \tilde{f}_{i}^{k} b \in B\right\} .
$$

Then the crystal graph $B$ satisfies the following properties.

Proposition 2.3. ([11, 12, 13])

(a) For all $i \in I$ and $b \in B$, we have

$$
\begin{aligned}
& \varphi_{i}(b)=\varepsilon_{i}(b)+\left\langle h_{i}, \operatorname{wt}(b)\right\rangle, \\
& \operatorname{wt}\left(\tilde{e}_{i} b\right)=\operatorname{wt}(b)+\alpha_{i}, \\
& \operatorname{wt}\left(\tilde{f}_{i} b\right)=\operatorname{wt}(b)-\alpha_{i} .
\end{aligned}
$$

(b) If $\tilde{e}_{i} b \in B$, then

$$
\varepsilon_{i}\left(\tilde{e}_{i} b\right)=\varepsilon_{i}(b)-1, \quad \varphi_{i}\left(\tilde{e}_{i} b\right)=\varphi_{i}(b)+1 .
$$

(c) If $\tilde{f}_{i} b \in B$, then

$$
\varepsilon_{i}\left(\tilde{f}_{i} b\right)=\varepsilon_{i}(b)+1, \quad \varphi_{i}\left(\tilde{f}_{i} b\right)=\varphi_{i}(b)-1 .
$$

Moreover, the crystal bases have extremely simple behavior with respect to taking the tensor product.

Proposition 2.4. (10, 11])

Let $M_{j}(j=1,2)$ be a $U_{q}(\mathfrak{g})$-module (or a $U_{q}^{\prime}(\mathfrak{g})$-module) in the category $\mathcal{O}_{\text {int }}$ and $\left(L_{j}, B_{j}\right)$ be its crystal basis. Set

$$
L=L_{1} \otimes_{\mathbf{A}} L_{2}, \quad B=B_{1} \times B_{2} .
$$


Then $(L, B)$ is a crystal basis of $M_{1} \otimes_{\mathbf{C}(q)} M_{2}$ with the Kashiwara operators on $B$ given by

$$
\begin{aligned}
& \tilde{e}_{i}\left(b_{1} \otimes b_{2}\right)= \begin{cases}\tilde{e}_{i} b_{1} \otimes b_{2} & \text { if } \varphi_{i}\left(b_{1}\right) \geq \varepsilon_{i}\left(b_{2}\right), \\
b_{1} \otimes \tilde{e}_{i} b_{2} & \text { if } \varphi_{i}\left(b_{1}\right)<\varepsilon_{i}\left(b_{2}\right),\end{cases} \\
& \tilde{f}_{i}\left(b_{1} \otimes b_{2}\right)= \begin{cases}\tilde{f}_{i} b_{1} \otimes b_{2} & \text { if } \varphi_{i}\left(b_{1}\right)>\varepsilon_{i}\left(b_{2}\right), \\
b_{1} \otimes \tilde{f}_{i} b_{2} & \text { if } \varphi_{i}\left(b_{1}\right) \leq \varepsilon_{i}\left(b_{2}\right) .\end{cases}
\end{aligned}
$$

As an immediate corollary, we obtain the following simple criterion for determining the maximal vectors in the tensor product of crystal graphs.

Corollary 2.5. ([10, 11])

(a) Let $M_{j}$ be a $U_{q}(\mathfrak{g})$-module (or a $U_{q}^{\prime}(\mathfrak{g})$-module) in the category $\mathcal{O}_{\text {int }}$ and let $\left(L_{j}, B_{j}\right)$ be a crystal basis of $M_{j}(j=1,2)$. Then $b_{1} \otimes b_{2} \in B_{1} \otimes B_{2}$ is a maximal vector (i.e., $\tilde{e}_{i}\left(b_{1} \otimes b_{2}\right)=0$ for all $i \in I$ ) if and only if $\tilde{e}_{i} b_{1}=0$ and $\left\langle h_{i}\right.$, wt $\left.b_{1}\right\rangle \geq \varepsilon_{i}\left(b_{2}\right)$ for all $i \in I$.

(b) Let $M_{j}$ be a $U_{q}(\mathfrak{g})$-module (or a $U_{q}^{\prime}(\mathfrak{g})$-module) in the category $\mathcal{O}_{\text {int }}$ and let $\left(L_{j}, B_{j}\right)$ be a crystal basis of $M_{j}(j=1, \cdots, N)$. Then the vector $b_{1} \otimes \cdots \otimes$ $b_{N} \in B_{1} \otimes \cdots \otimes B_{N}$ is a maximal vector if and only if $b_{1} \otimes \cdots \otimes b_{k}$ is a maximal vector for all $k=1, \cdots, N$.

The tensor product rule gives a very convenient combinatorial description of the action of Kashiwara operators on the multi-fold tensor product of crystal graphs. Let $M_{j}$ be a $U_{q}(\mathfrak{g})$-module (or a $U_{q}^{\prime}(\mathfrak{g})$-module) in the category $\mathcal{O}_{\text {int }}$ with a crystal basis $\left(L_{j}, B_{j}\right) \quad(j=1, \cdots, N)$. Fix an index $i \in I$ and consider a vector $b=b_{1} \otimes \cdots \otimes b_{N} \in B_{1} \otimes \cdots \otimes B_{N}$. To each $b_{j} \in B_{j} \quad(j=1, \cdots, N)$, we assign a sequence of -'s and +'s with as many -'s as $\varepsilon_{i}\left(b_{j}\right)$ followed by as many +'s as $\varphi_{i}\left(b_{j}\right)$ :

$$
\begin{aligned}
b & =b_{1} \otimes b_{2} \otimes \cdots \otimes b_{N} \\
& \longmapsto(\underbrace{-, \cdots,-}_{\varepsilon_{i}\left(b_{1}\right)}, \underbrace{+, \cdots,+}_{\varphi_{i}\left(b_{1}\right)}, \cdots \cdots, \underbrace{-, \cdots,-}_{\varepsilon_{i}\left(b_{N}\right)}, \underbrace{+, \cdots,+}_{\varphi_{i}\left(b_{N}\right)}) .
\end{aligned}
$$

In this sequence, we cancel out all the $(+,-)$-pairs to obtain a sequence of - 's followed by +'s:

$$
i-\operatorname{sgn}(b)=(-,-, \cdots,-,+,+, \cdots,+) .
$$

The sequence $i$-sgn $(b)$ is called the $i$-signature of $b$.

Now the tensor product rule tells that $\tilde{e}_{i}$ acts on $b_{j}$ corresponding to the right-most - in $i$-sgn $(b)$ and $\tilde{f}_{i}$ acts on $b_{k}$ corresponding to the left-most + in $i-\operatorname{sgn}(b)$ :

$$
\begin{aligned}
& \tilde{e}_{i} b=b_{1} \otimes \cdots \otimes \tilde{e}_{i} b_{j} \otimes \cdots \otimes b_{N}, \\
& \tilde{f}_{i} b=b_{1} \otimes \cdots \otimes \tilde{f}_{i} b_{k} \otimes \cdots \otimes b_{N} .
\end{aligned}
$$


We define $\tilde{e}_{i} b=0$ (resp. $\left.\tilde{f}_{i} b=0\right)$ if there is no $-($ resp. + ) in the $i$-signature of $b$.

We close this section with the existence and uniquenes theorem for crystal bases.

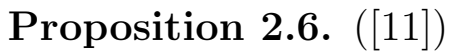

(a) Every $U_{q}(\mathfrak{g})$-module (resp. $U_{q}^{\prime}(\mathfrak{g})$-module) in the category $\mathcal{O}_{\text {int }}$ has a unique crystal basis. More precisely, let $V(\lambda)$ be the irreducible highest weight $U_{q}(\mathfrak{g})$-module (resp. $U_{q}^{\prime}(\mathfrak{g})$-module) with highest weight $\lambda \in P^{+}$(resp. $\quad \lambda \in$ $\left.\bar{P}^{+}\right)$and highest weight vector $u_{\lambda}$. Let $L(\lambda)$ be the free $\mathbf{A}$-submodule of $V(\lambda)$ spanned by the vectors of the form $\tilde{f}_{i_{1}} \cdots \tilde{f}_{i_{r}} u_{\lambda}\left(i_{k} \in I, r \in \mathbf{Z}_{\geq 0}\right)$ and set

$$
B(\lambda)=\left\{\tilde{f}_{i_{1}} \cdots \tilde{f}_{i_{r}} u_{\lambda} \in L(\lambda) / q L(\lambda)\right\} \backslash\{0\} .
$$

Then $(L(\lambda), B(\lambda))$ is a crystal basis of $V(\lambda)$ and every crystal basis of $V(\lambda)$ is isomorphic to $(L(\lambda), B(\lambda))$.

(b) Let $M$ be a $U_{q}(\mathfrak{g})$-module (resp. a $U_{q}^{\prime}(\mathfrak{g})$-module) in the category $\mathcal{O}_{\text {int }}$ and $(L, B)$ be its crystal basis. Define an automorphism of $U_{q}(\mathfrak{g})$ (resp. of $\left.U_{q}^{\prime}(\mathfrak{g})\right)$ by $\overline{e_{i}}=e_{i}, \overline{f_{i}}=f_{i}$, and $\overline{q^{h}}=q^{-h}$ for $i \in I, h \in P^{\vee}\left(\right.$ resp. $\left.h \in \bar{P}^{\vee}\right)$. Then there exists a unique $\mathbf{C}(q)$-basis $G=\{G(b) \mid b \in B\}$ of $M$ such that

$$
\overline{G(b)}=G(b), \quad G(b) \equiv b \quad(\bmod q L) \quad \text { for all } b \in B .
$$

Definition 2.7. The basis $G$ of $M$ given in Proposition 2.6 is called the global basis or the canonical basis of $M$ associated with the crystal graph $B$.

\section{Perfect CRystals}

By extracting properties of the crystal graphs, we define the notion of abstract crystals as follows (cf. 12, 13]).

Definition 3.1. An affine crystal (resp. classical crystal) is a set $B$ together with the maps wt $: B \rightarrow P$ (resp. wt $: B \rightarrow \bar{P}$ ), $\varepsilon_{i}: B \rightarrow \mathbf{Z} \cup\{-\infty\}$, $\varphi_{i}: B \rightarrow \mathbf{Z} \cup\{-\infty\}, \tilde{e}_{i}: B \rightarrow B \cup\{0\}$, and $\tilde{f}_{i}: B \rightarrow B \cup\{0\}$ satisfying the following conditions:

(i) for all $i \in I, b \in B$, we have

$$
\begin{aligned}
& \varphi_{i}(b)=\varepsilon_{i}(b)+\left\langle h_{i}, \operatorname{wt}(b)\right\rangle, \\
& \operatorname{wt}\left(\tilde{e}_{i} b\right)=\operatorname{wt}(b)+\alpha_{i}, \\
& \operatorname{wt}\left(\tilde{f}_{i} b\right)=\operatorname{wt}(b)-\alpha_{i} .
\end{aligned}
$$

(ii) if $\tilde{e}_{i} b \in B$, then

$$
\varepsilon_{i}\left(\tilde{e}_{i} b\right)=\varepsilon_{i}(b)-1, \quad \varphi_{i}\left(\tilde{e}_{i} b\right)=\varphi_{i}(b)+1 .
$$


(iii) if $\tilde{f}_{i} b \in B$, then

$$
\varepsilon_{i}\left(\tilde{f}_{i} b\right)=\varepsilon_{i}(b)+1, \quad \varphi_{i}\left(\tilde{f}_{i} b\right)=\varphi_{i}(b)-1 .
$$

(iv) $\tilde{f}_{i} b=b^{\prime}$ if and only if $b=\tilde{e}_{i} b^{\prime}$ for all $i \in I, b, b^{\prime} \in B$,

(v) if $\varepsilon_{i}(b)=-\infty$, then $\tilde{e}_{i} b=\tilde{f}_{i} b=0$.

The crystal graphs for $U_{q}(\mathfrak{g})$-modules (resp. $U_{q}^{\prime}(\mathfrak{g})$-modules) in the category $\mathcal{O}_{\text {int }}$ are affine crystals (resp. classical crystals).

Definition 3.2. Let $B_{1}$ and $B_{2}$ be (affine or classical) crystals. A morphism $\psi: B_{1} \rightarrow B_{2}$ of crystals is a map $\psi: B_{1} \cup\{0\} \rightarrow B_{2} \cup\{0\}$ satisfying the conditions:

(i) $\psi(0)=0$,

(ii) if $b \in B_{1}$ and $\psi(b) \in B_{2}$, then

$$
\operatorname{wt}(\psi(b))=\operatorname{wt}(b), \quad \varepsilon_{i}(\psi(b))=\varepsilon_{i}(b), \quad \varphi_{i}(\psi(b))=\varphi_{i}(b),
$$

(iii) if $b, b^{\prime} \in B_{1}, \psi(b), \psi\left(b^{\prime}\right) \in B_{2}$ and $\tilde{f}_{i} b=b^{\prime}$, then $\tilde{f}_{i} \psi(b)=\psi\left(b^{\prime}\right)$.

A morphism of crystals is said to be strict if it commutes with the Kashiwara operators $\tilde{e}_{i}$ and $\tilde{f}_{i}(i \in I)$.

Definition 3.3. The tensor product $B_{1} \otimes B_{2}$ of the crystals $B_{1}$ and $B_{2}$ is defined to be the set $B_{1} \times B_{2}$ whose crystal structure is given by

$$
\begin{aligned}
& \mathrm{wt}\left(b_{1} \otimes b_{2}\right)=\mathrm{wt}\left(b_{1}\right)+\mathrm{wt}\left(b_{2}\right), \\
& \varepsilon_{i}\left(b_{1} \otimes b_{2}\right)=\max \left(\varepsilon_{i}\left(b_{1}\right), \varepsilon_{i}\left(b_{2}\right)-\left\langle h_{i}, \operatorname{wt}\left(b_{1}\right)\right\rangle\right), \\
& \varphi_{i}\left(b_{1} \otimes b_{2}\right)=\max \left(\varphi_{i}\left(b_{2}\right), \varepsilon_{i}\left(b_{1}\right)+\left\langle h_{i}, \operatorname{wt}\left(b_{2}\right)\right\rangle\right), \\
& \tilde{e}_{i}\left(b_{1} \otimes b_{2}\right)= \begin{cases}\tilde{e}_{i} b_{1} \otimes b_{2} & \text { if } \varphi_{i}\left(b_{1}\right) \geq \varepsilon_{i}\left(b_{2}\right), \\
b_{1} \otimes \tilde{e}_{i} b_{2} & \text { if } \varphi_{i}\left(b_{1}\right)<\varepsilon_{i}\left(b_{2}\right),\end{cases} \\
& \tilde{f}_{i}\left(b_{1} \otimes b_{2}\right)= \begin{cases}\tilde{f}_{i} b_{1} \otimes b_{2} & \text { if } \varphi_{i}\left(b_{1}\right)>\varepsilon_{i}\left(b_{2}\right), \\
b_{1} \otimes \tilde{f}_{i} b_{2} & \text { if } \varphi_{i}\left(b_{1}\right) \leq \varepsilon_{i}\left(b_{2}\right) .\end{cases}
\end{aligned}
$$

Here, we denote $b_{1} \otimes b_{2}=\left(b_{1}, b_{2}\right)$ and use the convention that $b_{1} \otimes 0=0 \otimes b_{2}=0$.

We now define the notion of perfect crystals. Let $B$ be a classical crystal. For $b \in B$, we define

$$
\varepsilon(b)=\sum_{i} \varepsilon_{i}(b) \Lambda_{i} \quad \text { and } \quad \varphi(b)=\sum_{i} \varphi_{i}(b) \Lambda_{i} .
$$


Note that

$$
\mathrm{wt}(b)=\varphi(b)-\varepsilon(b) .
$$

For a positive integer $l>0$, set

$$
\bar{P}_{l}^{+}=\left\{\lambda \in \bar{P}^{+} \mid\langle c, \lambda\rangle=l\right\} .
$$

Definition 3.4. For $l \in \mathbf{Z}_{>0}$, we say that a finite classical crystal $\mathbf{B}$ is a perfect crystal of level $l$ if

(i) there is a finite dimensional $U_{q}^{\prime}(\mathfrak{g})$-module with a crystal basis whose crystal graph is isomorphic to $\mathbf{B}$,

(ii) $\mathbf{B} \otimes \mathbf{B}$ is connected,

(iii) there exists some $\lambda_{0} \in \bar{P}$ such that

$$
\operatorname{wt}(\mathbf{B}) \subset \lambda_{0}+\sum_{i \neq 0} \mathbf{Z}_{\leq 0} \alpha_{i}, \quad \#\left(\mathbf{B}_{\lambda_{0}}\right)=1,
$$

(iv) for any $b \in \mathbf{B}$, we have $\langle c, \varepsilon(b)\rangle \geq l$,

(v) for each $\lambda \in \bar{P}_{l}^{+}$, there exist unique $b^{\lambda} \in \mathbf{B}$ and $b_{\lambda} \in \mathbf{B}$ such that

$$
\varepsilon\left(b^{\lambda}\right)=\lambda, \quad \varphi\left(b_{\lambda}\right)=\lambda .
$$

A finite dimensional $U_{q}^{\prime}(\mathfrak{g})$-module $\mathbf{V}$ is called a perfect representation of level $l$ if it has a crystal basis $(L, B)$ such that $B$ is isomorphic to a perfect crystal of level $l$.

Remark. For a perfect crystal B, define

$$
\mathbf{B}^{\min }=\{b \in \mathbf{B} \mid\langle c, \varepsilon(b)\rangle=l\} .
$$

Then the maps $\varepsilon, \varphi: \mathbf{B}^{\min }=\{b \in B \mid\langle c, \varepsilon(b)\rangle=l\} \longrightarrow \bar{P}_{l}^{+}$are bijective.

In the following, we list some examples of perfect crystals of level 1 for the classical quantum affine algebras of type $A_{n}^{(1)}(n \geq 1), A_{2 n-1}^{(2)}(n \geq 3), D_{n}^{(1)}$ $(n \geq 4), A_{2 n}^{(2)}(n \geq 2), D_{n+1}^{(2)}(n \geq 2)$ and $B_{n}^{(1)}(n \geq 3)$.

Example 3.5. $A_{n}^{(1)}(n \geq 1)$ :

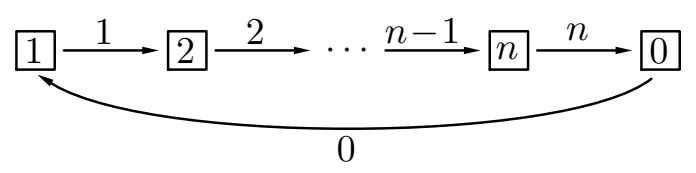

Here, we have

$$
b^{\Lambda_{i}}=i+1, \quad b_{\Lambda_{i}}=i \quad(i=0,1, \cdots, n) .
$$


Example 3.6. $A_{2 n-1}^{(2)}(n \geq 3)$ :

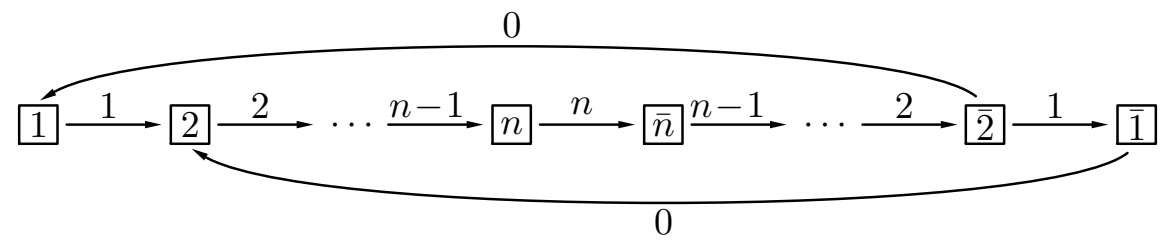

Here, we have

$$
b^{\Lambda_{0}}=1, b_{\Lambda_{0}}=\sqrt{1} ; \quad b^{\Lambda_{1}}=\sqrt{1}, b_{\Lambda_{1}}=1 .
$$

Example 3.7. $D_{n}^{(1)}(n \geq 4)$ :

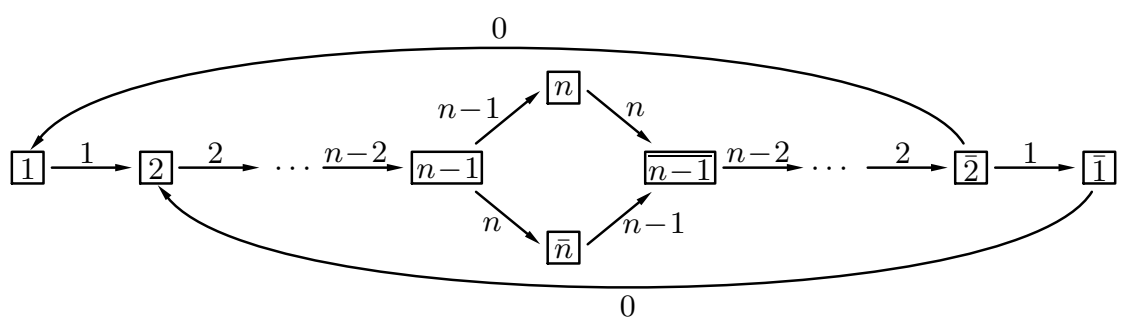

Here, we have

$$
\begin{aligned}
& b^{\Lambda_{0}}=1, b_{\Lambda_{0}}=\overline{1} ; \quad b^{\Lambda_{1}}=\overline{1}, b_{\Lambda_{1}}=1 \\
& b^{\Lambda_{n-1}}=n, b_{\Lambda_{n-1}}=\bar{n} ; \quad b^{\Lambda_{n}}=\bar{n}, b_{\Lambda_{n}}=n .
\end{aligned}
$$

Example 3.8. $A_{2 n}^{(2)}(n \geq 2)$ :

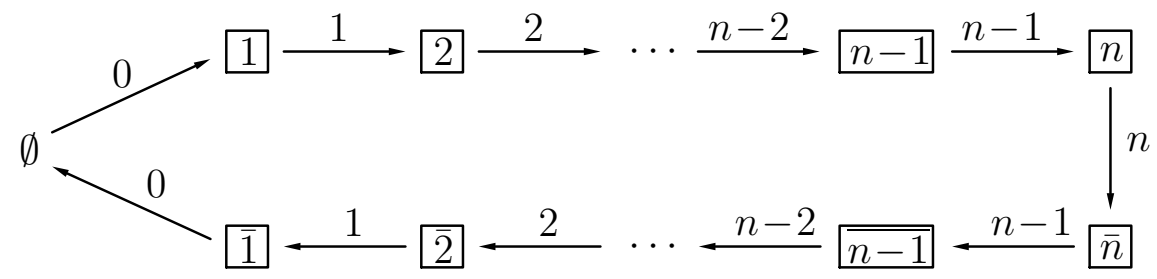

Here, we have

$$
b^{\Lambda_{0}}=\emptyset, \quad b_{\Lambda_{0}}=\emptyset .
$$

Example 3.9. $D_{n+1}^{(2)}(n \geq 2)$ : 


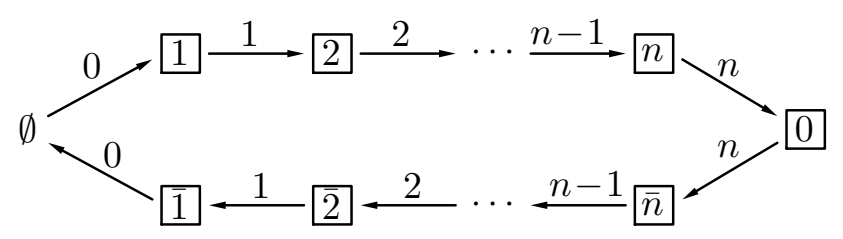

Here, we have

$$
b^{\Lambda_{0}}=\emptyset, \quad b_{\Lambda_{0}}=\emptyset ; \quad b^{\Lambda_{n}}=0, \quad b_{\Lambda_{n}}=0 .
$$

Example 3.10. $B_{n}^{(1)}(n \geq 3)$ :

$$
\begin{aligned}
& 0
\end{aligned}
$$

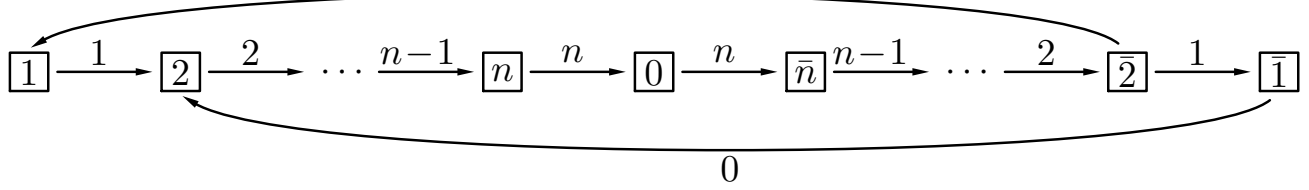

Here, we have

$$
\begin{array}{ll}
b^{\Lambda_{0}}=1, & b_{\Lambda_{0}}=\sqrt{1} ; \\
b^{\Lambda_{n}}=0, & b^{\Lambda_{1}}=\sqrt{\Lambda_{n}}=0 .
\end{array}
$$




\section{PATh Realization of CRYstal graphs}

Fix a positive integer $l>0$ and let $\mathbf{B}$ be a perfect crystal of level $l$. By definition, for any classical dominant integral weight $\lambda \in \bar{P}_{l}^{+}$, there exists a unique element $b_{\lambda} \in \mathbf{B}$ such that $\varphi\left(b_{\lambda}\right)=\lambda$. Set

$$
\mu=\lambda-\operatorname{wt}\left(b_{\lambda}\right)=\varepsilon\left(b_{\lambda}\right)
$$

and denote by $u_{\mu}$ the highest weight vector of the crystal graph $B(\mu)$. Then, using the fact that $\mathbf{B}$ is perfect, one can show that the vector $u_{\mu} \otimes b_{\lambda}$ is the unique maximal vector in $B(\mu) \otimes \mathbf{B}$. Moreover, we have:

Theorem 4.1. ([8]) Let $\mathbf{B}$ be a perfect crystal of level $l>0$. Then for any dominant integral weight $\lambda \in \bar{P}_{l}^{+}$, there exists a crystal isomorphism

$$
\Psi: B(\lambda) \stackrel{\sim}{\longrightarrow} B\left(\varepsilon\left(b_{\lambda}\right)\right) \otimes \mathbf{B} \quad \text { given by } \quad u_{\lambda} \longmapsto u_{\varepsilon\left(b_{\lambda}\right)} \otimes b_{\lambda},
$$

where $b_{\lambda}$ is the unique vector in $\mathbf{B}$ such that $\varphi\left(b_{\lambda}\right)=\lambda$.

Set

$$
\lambda_{0}=\lambda, \quad \lambda_{k+1}=\varepsilon\left(b_{\lambda_{k}}\right)
$$

and

$$
b_{0}=b_{\lambda}, \quad b_{k+1}=b_{\lambda_{k+1}} .
$$

By taking the composition of crystal isomorphism given in Theorem 4.1 repeatedly, we obtain a sequence of crystal isomorphisms

$$
B(\lambda) \stackrel{\sim}{\longrightarrow} B\left(\lambda_{1}\right) \otimes \mathbf{B} \stackrel{\sim}{\longrightarrow} B\left(\lambda_{2}\right) \otimes \mathbf{B} \otimes \mathbf{B} \stackrel{\sim}{\longrightarrow} \cdots \cdots
$$

given by

$$
u_{\lambda} \longmapsto u_{\lambda_{1}} \otimes b_{0} \longmapsto u_{\lambda_{2}} \otimes b_{1} \otimes b_{0} \longmapsto \cdots \cdots,
$$

which yields the infinite sequences

$$
\mathbf{w}_{\lambda}=\left(\lambda_{k}\right)_{k=0}^{\infty}=\left(\cdots, \lambda_{k+1}, \lambda_{k}, \cdots, \lambda_{1}, \lambda_{0}\right) \text { in }\left(\bar{P}_{l}^{+}\right)^{\infty}
$$

and

$$
\mathbf{p}_{\lambda}=\left(b_{k}\right)_{k=0}^{\infty}=\cdots \otimes b_{k+1} \otimes b_{k} \otimes \cdots \otimes b_{1} \otimes b_{0} \quad \text { in } \quad \mathbf{B}^{\otimes \infty} .
$$

Thus for each $k \geq 1$, we get a crystal isomorphism

$$
\Psi_{k}: B(\lambda) \stackrel{\sim}{\longrightarrow} B\left(\lambda_{k}\right) \otimes \mathbf{B}^{\otimes k}
$$

given by

$$
u_{\lambda} \longmapsto u_{\lambda_{k}} \otimes b_{k-1} \otimes \cdots \otimes b_{1} \otimes b_{0} .
$$

Since $\mathbf{B}$ is perfect, we have $\varphi\left(b_{j}\right)=\lambda_{j}$ and $\varepsilon\left(b_{j}\right)=\lambda_{j+1}$. It follows that the sequences

$$
\mathbf{w}_{\lambda}=\left(\lambda_{k}\right)_{k=0}^{\infty}=\left(\cdots, \lambda_{k+1}, \lambda_{k}, \cdots, \lambda_{1}, \lambda_{0}\right)
$$


and

$$
\mathbf{p}_{\lambda}=\left(b_{k}\right)_{k=0}^{\infty}=\cdots \otimes b_{k+1} \otimes b_{k} \otimes \cdots \otimes b_{1} \otimes b_{0}
$$

are periodic with the same period. That is, there is a positive integer $N>0$ such that $\lambda_{j+N}=\lambda_{j}, b_{j+N}=b_{j}$ for all $j=0,1, \cdots, N-1$.

\section{Definition 4.2.}

(a) The sequence $\mathbf{p}_{\lambda}=\left(b_{k}\right)_{k=0}^{\infty}=\cdots \otimes b_{k+1} \otimes b_{k} \otimes \cdots \otimes b_{1} \otimes b_{0}$ is called the ground-state path of weight $\lambda$.

(b) $\mathrm{A} \lambda$-path in $\mathbf{B}$ is a sequence $\mathbf{p}=(\mathbf{p}(k))_{k=0}^{\infty}=\cdots \otimes \mathbf{p}(k) \otimes \cdots \otimes \mathbf{p}(1) \otimes \mathbf{p}(0)$ such that $\mathbf{p}(k)=b_{k}$ for all $k \gg 0$.

Let $\mathbf{P}(\lambda)=\mathbf{P}(\lambda, \mathbf{B})$ be the set of all $\lambda$-paths in $\mathbf{B}$. We define the $U_{q}^{\prime}(\mathfrak{g})$ crystal structure on $\mathbf{P}(\lambda)$ as follows. Let $\mathbf{p}=(\mathbf{p}(k))_{k=0}^{\infty}$ be a $\lambda$-path in $\mathbf{P}(\lambda)$ and let $N>0$ be a positive integer such that $\mathbf{p}(k)=b_{k}$ for all $k \geq N$. For each $i \in I$, we define

$$
\begin{aligned}
& \overline{\mathrm{wt}}(\mathbf{p})=\lambda_{N}+\sum_{k=0}^{N-1} \overline{\mathrm{wt}} \mathbf{p}(k), \\
& \tilde{e}_{i} \mathbf{p}=\cdots \otimes \mathbf{p}(N+1) \otimes \tilde{e}_{i}(\mathbf{p}(N) \otimes \cdots \otimes \mathbf{p}(0)), \\
& \tilde{f}_{i} \mathbf{p}=\cdots \otimes \mathbf{p}(N+1) \otimes \tilde{f}_{i}(\mathbf{p}(N) \otimes \cdots \otimes \mathbf{p}(0)), \\
& \varepsilon_{i}(\mathbf{p})=\max \left(\varepsilon_{i}\left(\mathbf{p}^{\prime}\right)-\varphi_{i}\left(b_{N}\right), 0\right), \\
& \varphi_{i}(\mathbf{p})=\varphi_{i}\left(\mathbf{p}^{\prime}\right)+\max \left(\varphi_{i}\left(b_{N}\right)-\varepsilon_{i}\left(\mathbf{p}^{\prime}\right), 0\right),
\end{aligned}
$$

where $\mathbf{p}^{\prime}=\mathbf{p}(N-1) \otimes \cdots \otimes \mathbf{p}(1) \otimes \mathbf{p}(0)$.

Then we have the path realization of the classical crystal $B(\lambda)$ :

Theorem 4.3. ([8])

(a) The maps $\overline{w t}: \mathbf{P}(\lambda) \rightarrow \bar{P}, \tilde{e}_{i}, \tilde{f}_{i}: \mathbf{P}(\lambda) \rightarrow \mathbf{P}(\lambda) \cup\{0\}, \varepsilon_{i}, \varphi_{i}: \mathbf{P}(\lambda) \rightarrow \mathbf{Z}$ define a classical crystal structure on $\mathbf{P}(\lambda)$.

(b) There exists an isomorphism of classical crystals

$$
\Psi: B(\lambda) \stackrel{\sim}{\longrightarrow} \mathbf{P}(\lambda) \text { given by } u_{\lambda} \longmapsto \mathbf{p}_{\lambda} \text {. }
$$

In the following examples, we list the ground-state paths for basic representations of quantum affine algebras and illustrate the top part of their crystal graphs for some small ranks.

Example 4.4. $A_{n}^{(1)}(n \geq 1)$

(a) Ground-state paths 


$$
\mathbf{p}_{\Lambda_{i}}=\left(\mathbf{p}_{\Lambda_{i}}(k)\right)_{k=0}^{\infty}=(\cdots, 1,2, \cdots, n, 0,1,2, \cdots, i)
$$


(b) Crystal graph $\mathbf{B}\left(\Lambda_{0}\right)$ for $A_{2}^{(1)}$

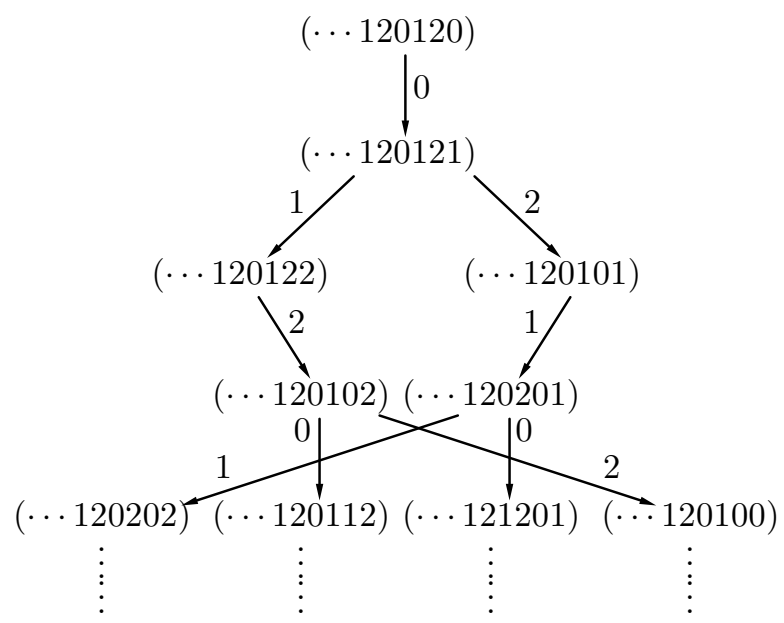

Example 4.5. $A_{2 n-1}^{(2)}(n \geq 3)$

(a) Ground-state paths

$$
\begin{aligned}
& \mathbf{p}_{\Lambda_{0}}=\left(\mathbf{p}_{\Lambda_{0}}(k)\right)_{k=0}^{\infty}=(\cdots, 1, \overline{1}, 1, \overline{1}, 1, \overline{1}), \\
& \mathbf{p}_{\Lambda_{1}}=\left(\mathbf{p}_{\Lambda_{1}}(k)\right)_{k=0}^{\infty}=(\cdots, \overline{1}, 1, \overline{1}, 1, \overline{1}, 1) .
\end{aligned}
$$

(b) Crystal graph $\mathbf{B}\left(\Lambda_{0}\right)$ for $A_{5}^{(2)}$

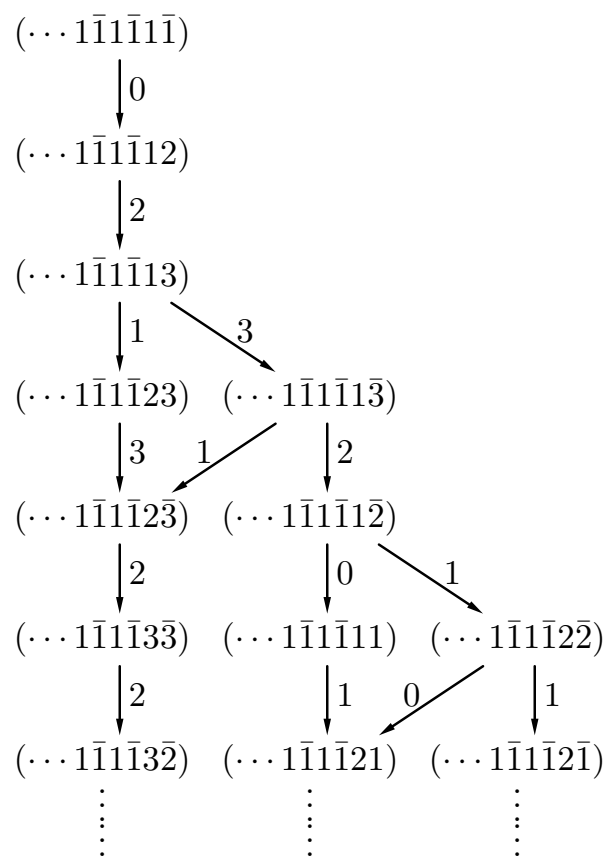


Example 4.6. $D_{n}^{(1)}(n \geq 4)$

(a) Ground-state paths

$$
\begin{aligned}
& \mathbf{p}_{\Lambda_{0}}=\left(\mathbf{p}_{\Lambda_{0}}(k)\right)_{k=0}^{\infty}=(\cdots, 1, \overline{1}, 1, \overline{1}, 1, \overline{1}), \\
& \mathbf{p}_{\Lambda_{1}}=\left(\mathbf{p}_{\Lambda_{1}}(k)\right)_{k=0}^{\infty}=(\cdots, \overline{1}, 1, \overline{1}, 1, \overline{1}, 1), \\
& \mathbf{p}_{\Lambda_{n-1}}=\left(\mathbf{p}_{\Lambda_{n-1}}(k)\right)_{k=0}^{\infty}=(\cdots, n, \bar{n}, n, \bar{n}, n, \bar{n}), \\
& \mathbf{p}_{\Lambda_{n}}=\left(\mathbf{p}_{\Lambda_{n}}(k)\right)_{k=0}^{\infty}=(\cdots, \bar{n}, n, \bar{n}, n, \bar{n}, n),
\end{aligned}
$$

(b) Crystal graph $\mathbf{B}\left(\Lambda_{0}\right)$ for $D_{4}^{(1)}$

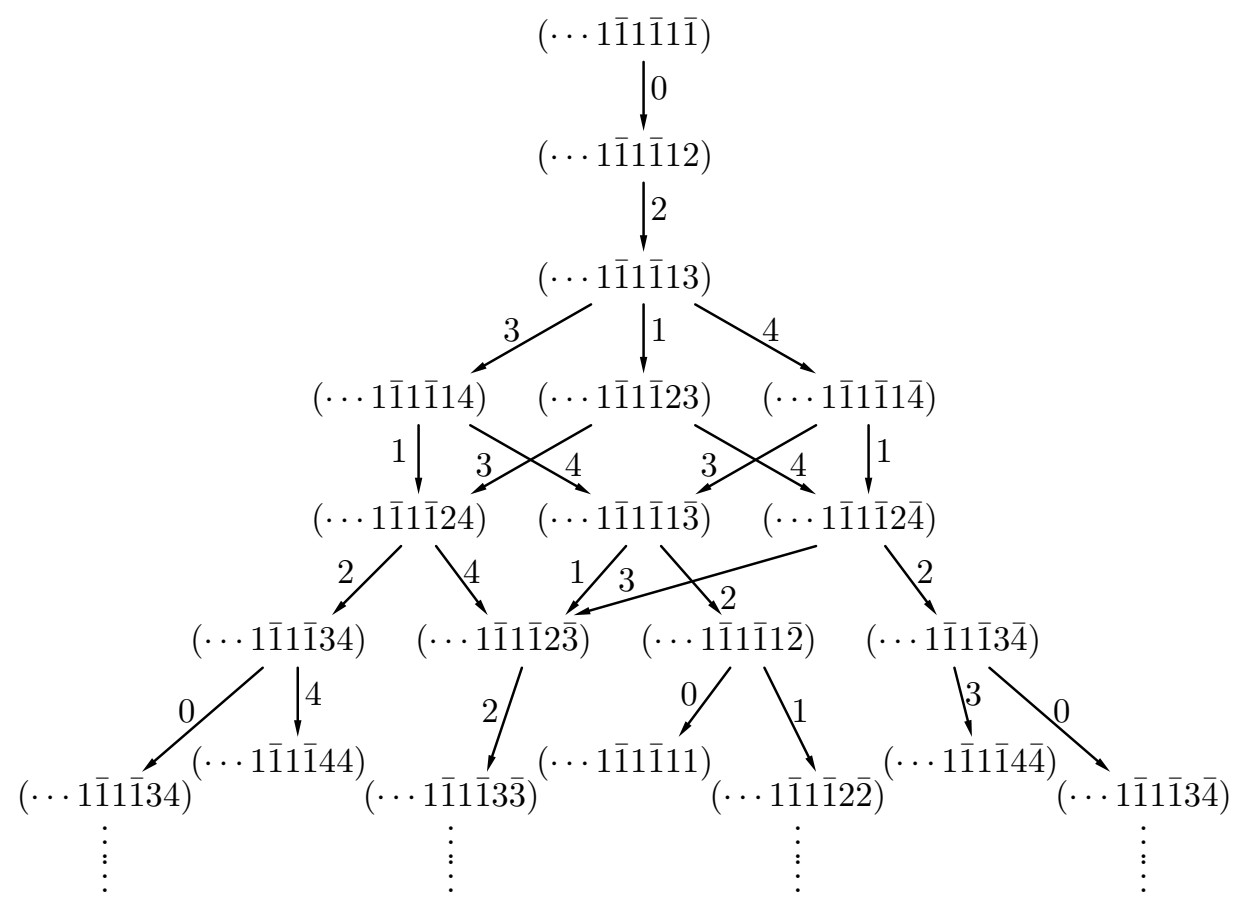

Example 4.7. $A_{2 n}^{(2)}(n \geq 2)$

(a) Ground-state paths

$$
\mathbf{p}_{\Lambda_{0}}=\left(\mathbf{p}_{\Lambda_{0}}(k)\right)_{k=0}^{\infty}=(\cdots, \emptyset, \emptyset, \emptyset, \emptyset, \emptyset, \emptyset)
$$

(b) Crystal graph $\mathbf{B}\left(\Lambda_{0}\right)$ for $A_{4}^{(2)}$ 


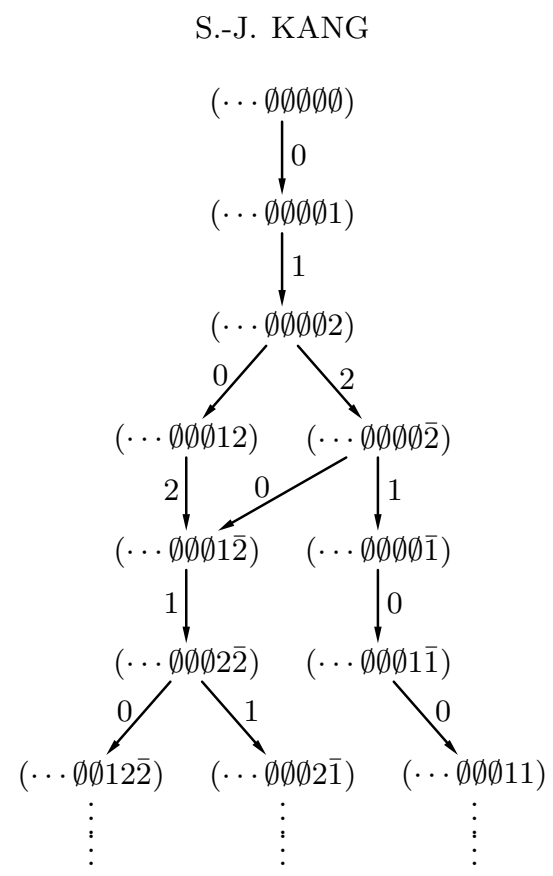

Example 4.8. $D_{n+1}^{(2)}(n \geq 2)$.

(a) Ground-state paths

$$
\begin{aligned}
& \mathbf{p}_{\Lambda_{0}}=\left(\mathbf{p}_{\Lambda_{0}}(k)\right)_{k=0}^{\infty}=(\cdots, \emptyset, \emptyset, \emptyset, \emptyset, \emptyset, \emptyset) \\
& \mathbf{p}_{\Lambda_{n}}=\left(\mathbf{p}_{\Lambda_{n}}(k)\right)_{k=0}^{\infty}=(\cdots, 0,0,0,0,0,0)
\end{aligned}
$$

(b) Crystal graph $\mathbf{B}\left(\Lambda_{0}\right)$ for $D_{3}^{(2)}$

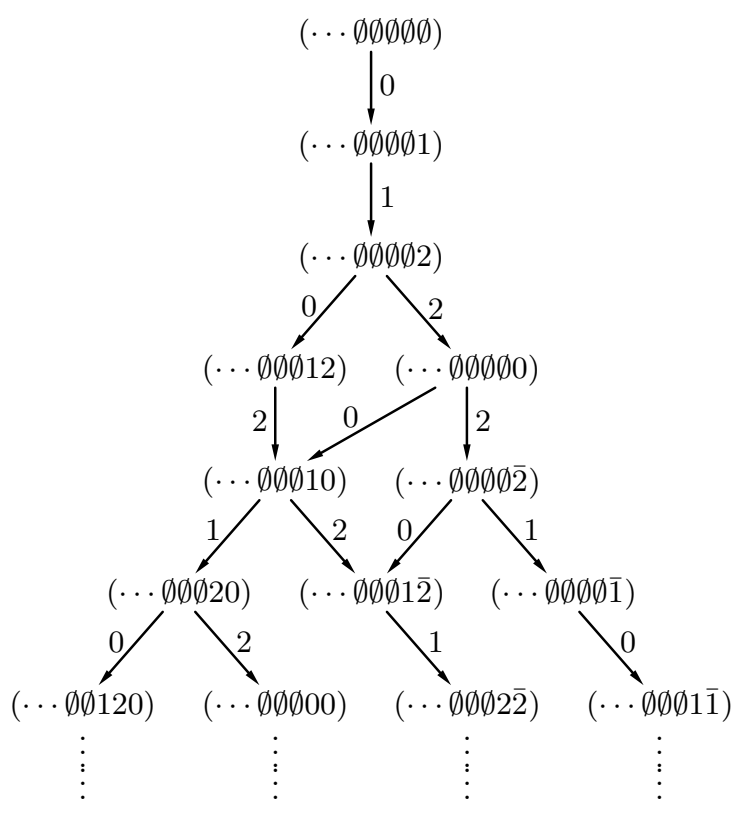


Example 4.9. $B_{n}^{(1)}(n \geq 3)$

(a) Ground-state paths

$$
\begin{aligned}
& \mathbf{p}_{\Lambda_{0}}=\left(\mathbf{p}_{\Lambda_{0}}(k)\right)_{k=0}^{\infty}=(\cdots, 1, \overline{1}, 1, \overline{1}, 1, \overline{1}), \\
& \mathbf{p}_{\Lambda_{1}}=\left(\mathbf{p}_{\Lambda_{1}}(k)\right)_{k=0}^{\infty}=(\cdots, \overline{1}, 1, \overline{1}, 1, \overline{1}, 1), \\
& \mathbf{p}_{\Lambda_{n}}=\left(\mathbf{p}_{\Lambda_{n}}(k)\right)_{k=0}^{\infty}=(\cdots, 0,0,0,0,0,0)
\end{aligned}
$$

(b) Crystal graph $\mathbf{B}\left(\Lambda_{0}\right)$ for $B_{3}^{(1)}$

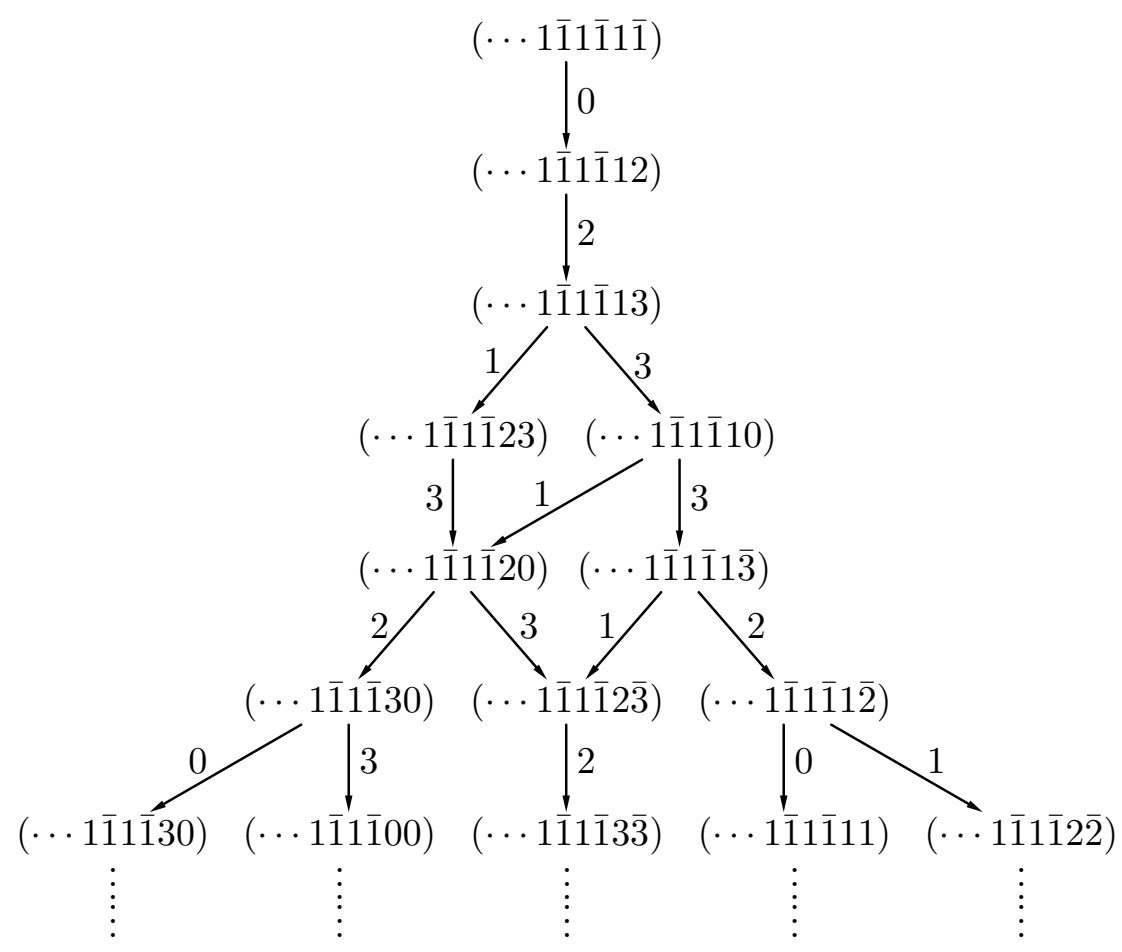


(c) Crystal graph $\mathbf{B}\left(\Lambda_{3}\right)$ for $B_{3}^{(1)}$

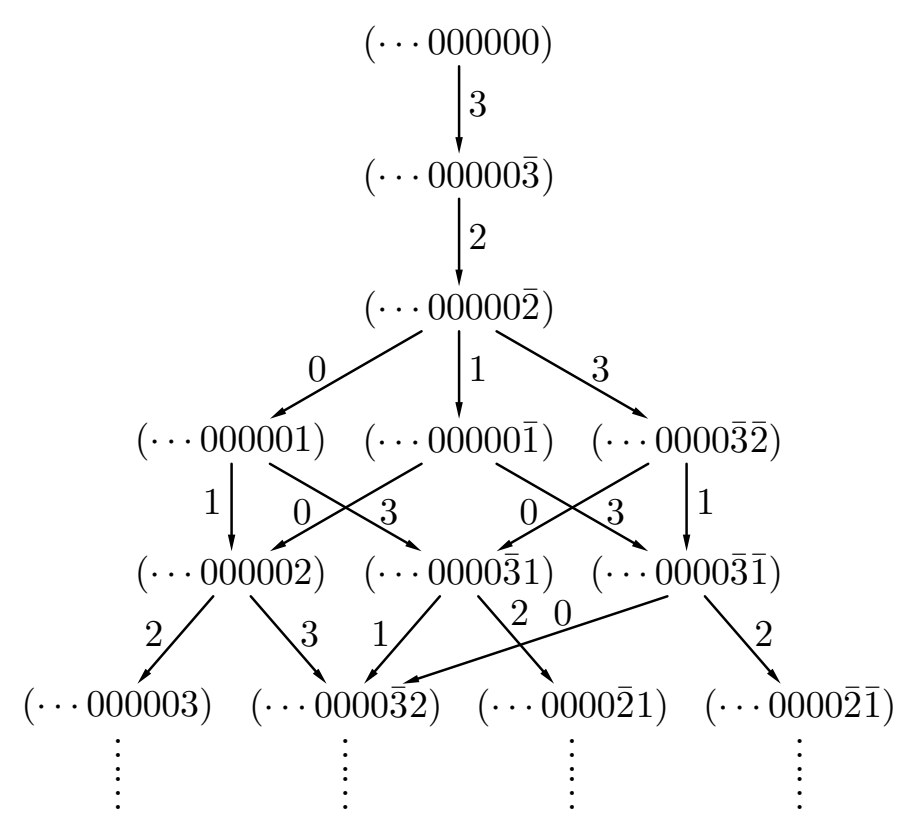

\section{YOUNG WALLS}

The purpose of this paper is to give a realization of crystal graph $B(\lambda)$ for the basic representations of quantum affine algebras in terms of new combinatorial objects which we call the Young walls. In this section, we explain the notion of Young walls. The Young walls are built of colored blocks of three different shapes:

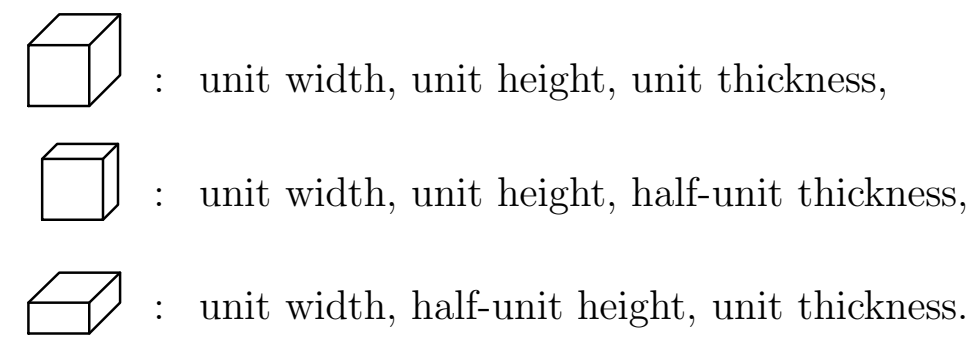

With these colored blocks, we will build a wall of thickness less than or equal to one unit which extends infinitely to the left like playing with the LEGO blocks. Given a dominant integral weight $\lambda$ of level 1, we fix a frame called the ground-state wall of weight $\lambda$, and build the Young walls on this frame. For each type of classical quantum affine algebras, we use different sets of colored blocks and ground-state walls, as are described in the following. 
(a) $A_{n}^{(1)}(n \geq 1)$

$j(j=0,1, \cdots, n):$ unit width, unit height, unit thickness,

$Y_{\Lambda_{i}}=\nearrow \quad(i=0,1, \cdots, n)$.

(b) $A_{2 n-1}^{(2)}(n \geq 3)$

0 1 : unit width, unit height, half-unit thickness,

$j(j=2, \cdots, n):$ unit width, unit height, unit thickness,

$Y_{\Lambda_{0}}=$\begin{tabular}{|l|l|l|l|}
\hline 0 & 1 & 0 & 1 \\
\hline
\end{tabular}

$Y_{\Lambda_{1}}=$\begin{tabular}{|l|l|l|l|}
\hline 1 & 0 & 1 & 0 \\
\hline
\end{tabular}

(c) $D_{n}^{(1)}(n \geq 4)$

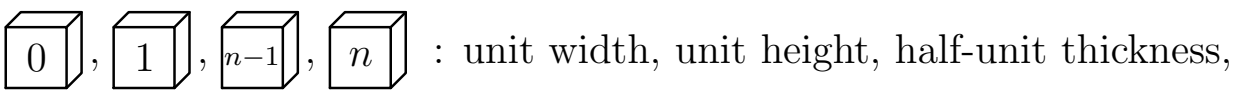

$j(j=2, \cdots, n-2):$ unit width, unit height, unit thickness,

$Y_{\Lambda_{0}}=$\begin{tabular}{|l|l|l|l|}
\hline 0 & 1 & 0 & 1 \\
\hline
\end{tabular}

$Y_{\Lambda_{1}}=$\begin{tabular}{|l|l|l|l|}
\hline 1 & 0 & 1 & 0 \\
\hline
\end{tabular}

$Y_{\Lambda_{n-1}}=$\begin{tabular}{|l|l|l|l|}
\hline$n-1$ & $n$ & $n-1$ & $n$ \\
\hline
\end{tabular}

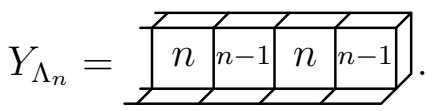


(d) $A_{2 n}^{(2)}(n \geq 2)$

0 : unit width, half-unit height, unit thickness,

$j(j=1, \cdots, n):$ unit width, unit height, unit thickness,

$Y_{\Lambda_{0}}=$\begin{tabular}{ll|l|l|l|}
\hline I & 0 & 0 & 0 & 0 \\
\hline
\end{tabular}

(e) $D_{n+1}^{(2)}(n \geq 2)$

D, : unit width, half-unit height, unit thickness,

$j(j=1, \cdots, n-1):$ unit width, unit height, unit thickness,

$Y_{\Lambda_{0}}=$\begin{tabular}{ll|l|l|l|}
\hline I & 0 & 0 & 0 & 0 \\
\hline
\end{tabular}

$Y_{\Lambda_{n}}=$\begin{tabular}{lllll}
\hline \\
\hline
\end{tabular}

(f) $B_{n}^{(1)}(n \geq 3)$

0 , 1 : unit width, unit height, half-unit thickness,

: unit width, half-unit height, unit thickness,

$j(j=2, \cdots, n-1):$ unit width, unit height, unit thickness,

$Y_{\Lambda_{0}}=$\begin{tabular}{|l|l|l|l|}
\hline 0 & 1 & 0 & 1 \\
\hline
\end{tabular}

$Y_{\Lambda_{1}}=$\begin{tabular}{|l|l|l|l|}
\hline 1 & 0 & 1 & 0 \\
\hline
\end{tabular}

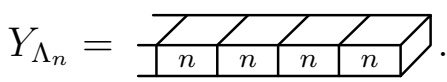

For convenience, we will use the following notations: 


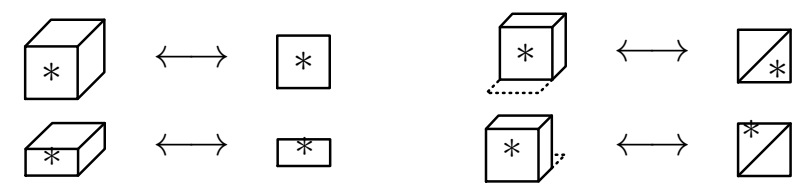

For example, we have

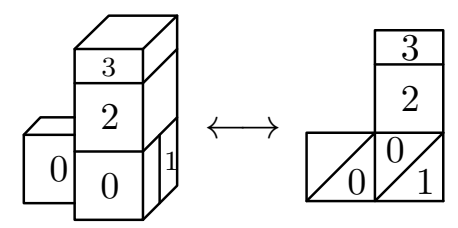

The rules for building the walls are given as follows:

1. The walls must be built on top of the ground-state walls.

2. The colored blocks should be stacked in columns. No block can be placed on top of a column of half-unit thickness.

3. Except for the right-most column, there should be no free space to the right of any block.

4. The colored blocks should be stacked in the patterns given below.

(a) $A_{n}^{(1)}(n \geq 1)$

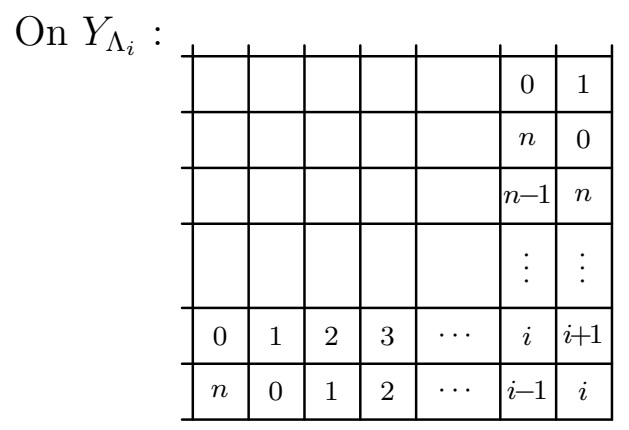

(b) $A_{2 n-1}^{(2)}(n \geq 3)$

On $Y_{\Lambda_{0}}$ :

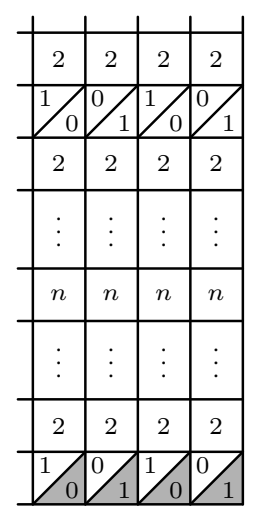

On $Y_{\Lambda_{1}}$ :

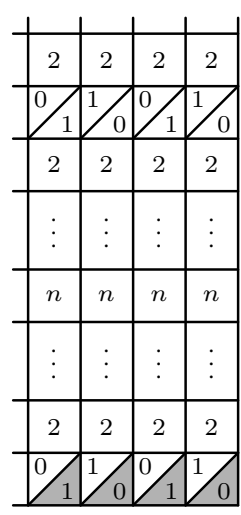


(c) $D_{n}^{(1)}(n \geq 4)$

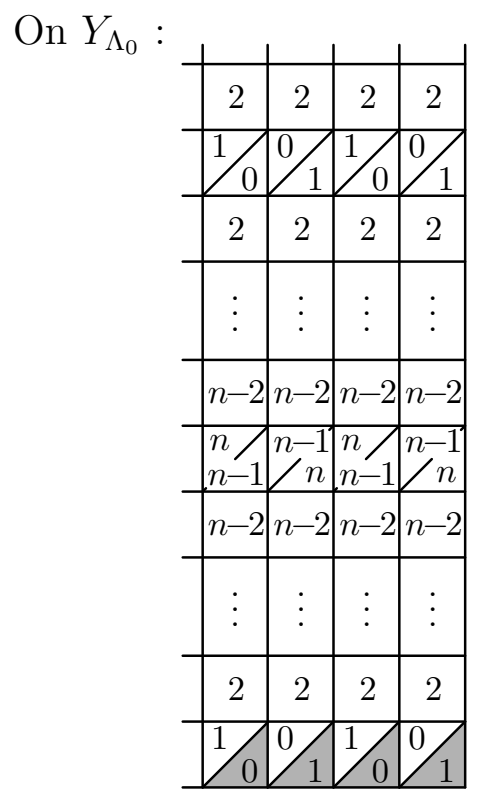

On $Y_{\Lambda_{1}}$ :

\begin{tabular}{|c|c|c|c|}
\hline 2 & 2 & 2 & 2 \\
\hline $0 / 1$ & $1 / 0$ & $0 / 1$ & $1 / 0$ \\
\hline 2 & 2 & 2 & 2 \\
\hline$\vdots$ & $\vdots$ & $\cdot$ & \\
\hline$n-2$ & $n-2$ & $n-2$ & $n-2$ \\
\hline $\begin{array}{l}n / 1 \\
n-1\end{array}$ & $\begin{array}{l}n-1 \\
/ n\end{array}$ & $\begin{array}{l}n / \\
n-1\end{array}$ & $\begin{array}{l}n-1 \\
\angle n\end{array}$ \\
\hline$n-2$ & $n-2$ & $n-2$ & $n-2$ \\
\hline : & . & : & \\
\hline 2 & 2 & 2 & 2 \\
\hline & & & 40 \\
\hline
\end{tabular}

On $Y_{\Lambda_{n-1}}$ :

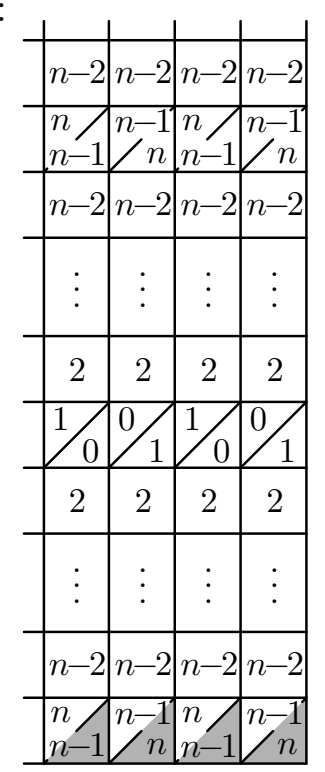

On $Y_{\Lambda_{n}}$ :

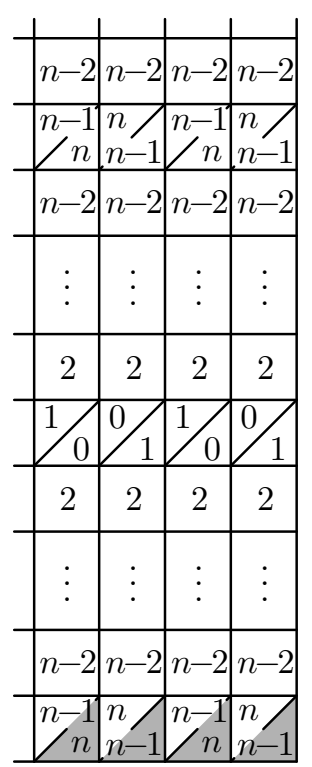


(d) $A_{2 n}^{(2)}(n \geq 2)$

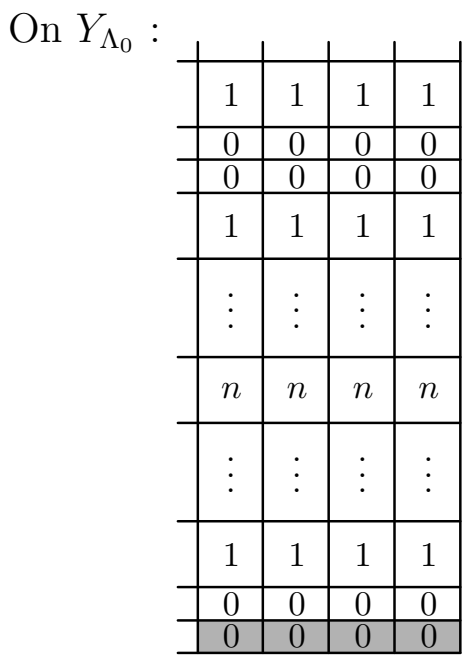

(e) $D_{n+1}^{(2)}(n \geq 2)$

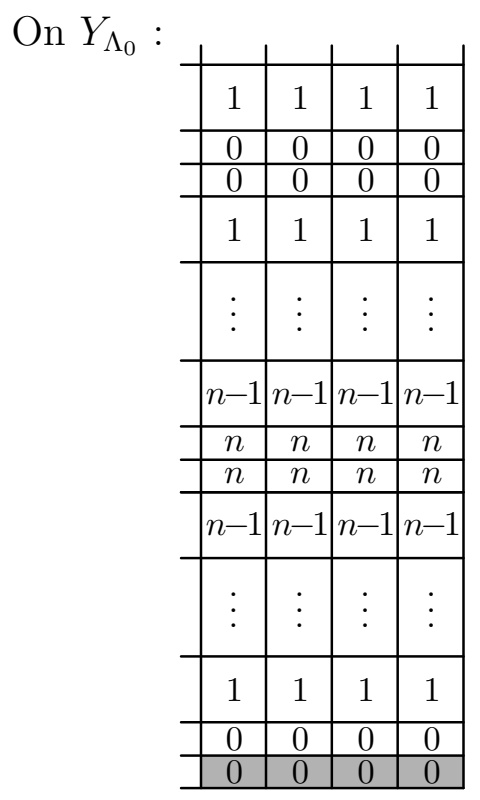

On $Y_{\Lambda_{n}}$ :

\begin{tabular}{c|c|c|c|}
\hline & & & \\
\hline$n-1$ & $n-1$ & $n-1$ & $n-1$ \\
\hline$n$ & $n$ & $n$ & $n$ \\
\hline$n$ & $n$ & $n$ & $n$ \\
\hline$n-1$ & $n-1$ & $n-1$ & $n-1$ \\
\hline$\vdots$ & $\vdots$ & $\vdots$ & $\vdots$ \\
\hline 1 & 1 & 1 & 1 \\
\hline 0 & 0 & 0 & 0 \\
\hline 0 & 0 & 0 & 0 \\
\hline 1 & 1 & 1 & 1 \\
\hline$\vdots$ & $\vdots$ & $\vdots$ & $\vdots$ \\
\hline$n-1$ & $n-1$ & $n-1$ & $n-1$ \\
\hline$n$ & $n$ & $n$ & $n$ \\
\hline$n$ & $n$ & $n$ & $n$ \\
\hline
\end{tabular}


(f) $B_{n}^{(1)}(n \geq 3)$

On $Y_{\Lambda_{0}}$ :

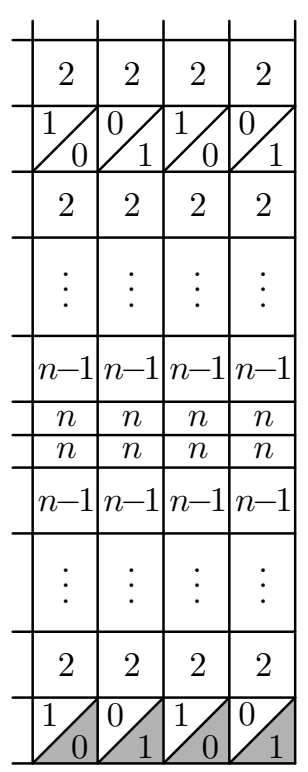

On $Y_{\Lambda_{1}}$ :

\begin{tabular}{|c|c|c|c|}
\hline & & & \\
\hline 2 & 2 & 2 & 2 \\
\hline $0 / 1$ & $1 / 0$ & $0 / 1$ & \\
\hline 2 & 2 & 2 & 2 \\
\hline & 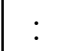 & 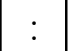 & \\
\hline$n-1$ & $n-1$ & $n-1$ & $n-1$ \\
\hline $\bar{n}$ & $n$ & $n$ & $\bar{n}$ \\
\hline$n$ & $n$ & $n$ & $n$ \\
\hline$n-1$ & $n-1$ & $n-1$ & $n-1$ \\
\hline . & . & : & \\
\hline 2 & 2 & 2 & 2 \\
\hline 07 & & 0 & 17 \\
\hline
\end{tabular}

On $Y_{\Lambda_{n}}$ :

\begin{tabular}{c|c|c|c|} 
& & & \\
\hline$n-1$ & $n-1$ & $n-1$ & $n-1$ \\
\hline$n$ & $n$ & $n$ & $n$ \\
\hline$n$ & $n$ & $n$ & $n$ \\
\hline$n-1$ & $n-1$ & $n-1$ & $n-1$ \\
\hline$\vdots$ & $\vdots$ & $\vdots$ & $\vdots$ \\
\hline 2 & 2 & 2 & 2 \\
\hline $1 / 0$ & 0 & $1 / 0$ & $0 / 1$ \\
\hline 2 & 2 & 2 & 2 \\
\hline$\vdots$ & $\vdots$ & $\vdots$ & $\vdots$ \\
\hline$n-1$ & $n-1$ & $n-1$ & $n-1$ \\
\hline$n$ & $n$ & $n$ & $n$ \\
\hline$n$ & $n$ & $n$ & $n$ \\
\hline
\end{tabular}




\section{Definition 5.1.}

(a) A wall built on the ground-state wall $Y_{\Lambda_{i}}$ following the rules listed above is called a Young wall on the ground-state $\Lambda_{i}$ if the heights of its columns are weakly decreasing as we proceed from the right to the left.

(b) A column in a Young wall is called a full column if its height is a multiple of the unit length and its top is of unit thickness.

(c) For the classical quantum affine algebras of type $A_{2 n-1}^{(2)}(n \geq 3), D_{n}^{(1)}$ $(n \geq 4), A_{2 n}^{(2)}(n \geq 2), D_{n+1}^{(2)}(n \geq 2)$ and $B_{n}^{(1)}(n \geq 3)$, a Young wall is said to be proper if none of the full columns have the same height.

(d) For the quantum affine algebras of type $A_{n}^{(1)}(n \geq 1)$, every Young wall is defined to be proper.

Example 5.2. Consider the Young wall $Y=\left(y_{k}\right)_{k=0}^{\infty}=\left(\cdots, y_{k}, \cdots, y_{1}, y_{0}\right)$ for $B_{3}^{(1)}$ built on the ground-state wall $Y_{\Lambda_{0}}$ :

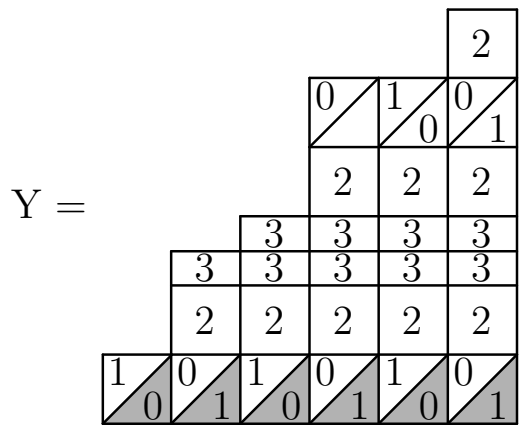

The columns $y_{0}, y_{1}, y_{3} y_{5}$ are full columns and $y_{2}, y_{4}$ are not full. Hence $Y$ is a proper Young wall.

On the other hand,

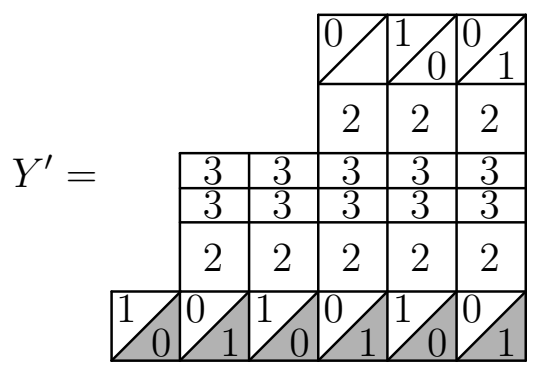

is not a proper Young wall because the full columns $y_{0}^{\prime}$ and $y_{1}^{\prime}$ (also $y_{3}^{\prime}$ and $y_{4}^{\prime}$ ) have the same height. 


\section{THE CRYstal STRUCTURE}

Let $\lambda$ be a dominant integral weight of level 1 and let $\mathbf{F}(\lambda)$ denote the set of all proper Young walls built on the ground-state $Y_{\lambda}$. In this section, we define an affine crystal structure on $\mathbf{F}(\lambda)$. The action of Kashiwara operators are defined using the $i$-signatures of proper Young walls in a similar way to playing the Tetris game.

\section{Definition 6.1.}

(a) A block of color $i$ in a proper Young wall is called a removable $i$-block if the wall remains a proper Young wall after removing the block. A column in a proper Young wall is called $i$-removable if the top of that column is a removable $i$-block.

(b) A place in a proper Young wall where one may add an $i$-block to obtain another proper Young wall is called an $i$-admissible slot. A column in a proper Young wall is called $i$-admissible if the top of that column is an $i$-admissible slot.

Example 6.2. In the following figure, we consider a proper Young wall for $B_{3}^{(1)}$ built on the ground-state wall $Y_{\Lambda_{0}}$ and indicate all the removable blocks and admissible slots.

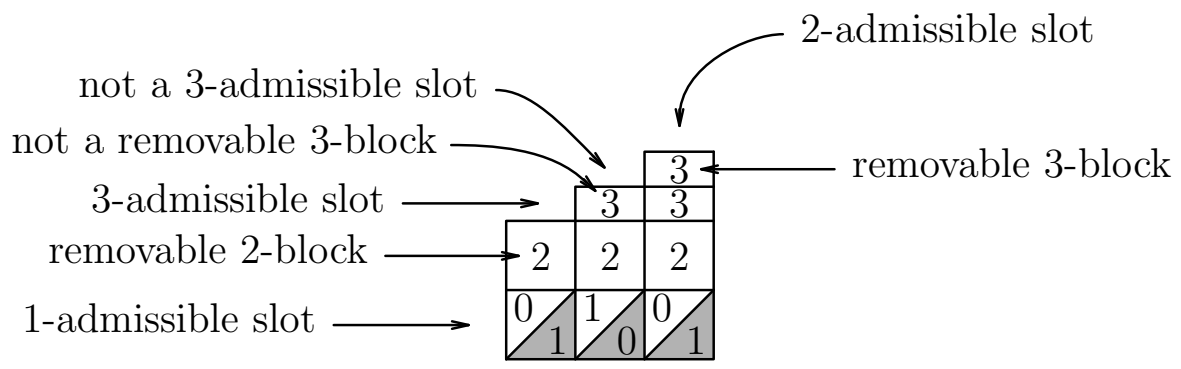

We now define the action of Kashiwara operators $\tilde{e}_{i}, \tilde{f}_{i}(i \in I)$ on $\mathbf{F}(\lambda)$. Fix $i \in I$ and let $Y=\left(y_{k}\right)_{k=0}^{\infty} \in \mathbf{F}(\lambda)$ be a proper Young wall.

1. To each column $y_{k}$ of $Y$, we assign its $i$-signature as follows:

(a) we assign - - if the column $y_{k}$ is twice $i$-removable;

(b) we assign - if the column is once $i$-removable, but not $i$-admissible (the $i$-block may be of unit height or of half-unit height);

(c) we assign -+ if the column is once $i$-removable and once $i$-admissible (the $i$-block will be of half-unit height in this case);

(d) we assign + if the column is once $i$-admissible, but not $i$-removable (the $i$-block may be of unit height or of half-unit height);

(e) we assign ++ if the column is twice $i$-admissible (the $i$-block will be of half-unit height in this case). 
2. From the (infinite) sequence of + 's and -'s, cancel out every $(+,-)$-pair to obtain a finite sequence of -'s followed by + 's, reading from left to right. This sequence is called the $i$-signature of the proper Young wall $Y$.

3. We define $\tilde{e}_{i} Y$ to be the proper Young wall obtained from $Y$ by removing the $i$-block corresponding to the right-most - in the $i$-signature of $Y$. We define $\tilde{e}_{i} Y=0$ if there exists no - in the $i$-signature of $Y$.

4. We define $\tilde{f}_{i} Y$ to be the proper Young wall obtained from $Y$ by adding an $i$-block to the column corresponding to the left-most + in the $i$-signature of $Y$. We define $\tilde{f}_{i} Y=0$ if there exists no + in the $i$-signature of $Y$.

We also define the maps

$$
\text { wt }: \mathbf{F}(\lambda) \longrightarrow P, \quad \varepsilon_{i}: \mathbf{F}(\lambda) \longrightarrow \mathbf{Z}, \quad \varphi_{i}: \mathbf{F}(\lambda) \longrightarrow \mathbf{Z}
$$

by

$$
\begin{aligned}
\operatorname{wt}(Y) & =\lambda-\sum_{i \in I} k_{i} \alpha_{i}, \\
\varepsilon_{i}(Y) & =\text { the number of }- \text { 's in the } i \text {-signature of } Y, \\
\varphi_{i}(Y) & =\text { the number of }+ \text { 's in the } i \text {-signature of } Y,
\end{aligned}
$$

where $k_{i}$ is the number of $i$-blocks in $Y$ that have been added to the groundstate wall $Y_{\lambda}$.

Then it is straightforward to verify that the following theorem holds.

Theorem 6.3. The maps wt $: \mathbf{F}(\lambda) \rightarrow P, \tilde{e}_{i}, \tilde{f}_{i}: \mathbf{F}(\lambda) \rightarrow \mathbf{F}(\lambda) \cup\{0\}$ and $\varepsilon_{i}, \varphi_{i}: \mathbf{F}(\lambda) \rightarrow \mathbf{Z}$ define an affine crystal structure on the set $\mathbf{F}(\lambda)$ of all proper Young walls.

Let $\delta$ be the null root for the quantum affine algebra $U_{q}(\mathfrak{g})$ and write

$$
\begin{cases}\delta=a_{0} \alpha_{0}+a_{1} \alpha_{1}+\cdots+a_{n} \alpha_{n} & \text { for } \mathfrak{g}=A_{n}^{(1)}, \cdots, B_{n}^{(1)} \\ 2 \delta=a_{0} \alpha_{0}+a_{1} \alpha_{1}+\cdots+a_{n} \alpha_{n} & \text { for } \mathfrak{g}=D_{n+1}^{(2)}\end{cases}
$$

The part of a column consisting of $a_{0}$-many 0 -blocks, $a_{1}$-many 1 -blocks, $\cdots$, $a_{n}$-many $n$-blocks in some cyclic order is called a $\delta$-column.

\section{Example 6.4.}

1. The following are $\delta$-columns for $B_{3}^{(1)}$. 


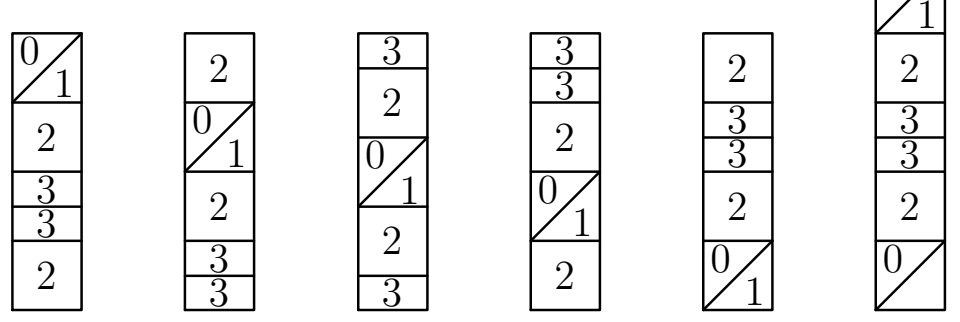

2. The following are $\delta$-columns for $D_{3}^{(2)}$.

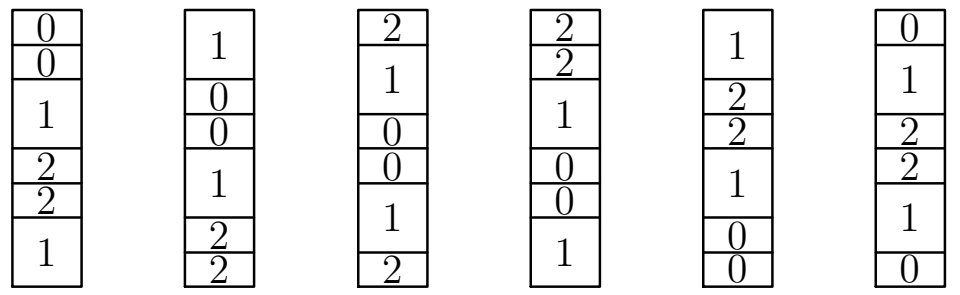

\section{Definition 6.5.}

(a) A column in a proper Young wall is said to contain a removable $\delta$ if we may remove a $\delta$-column from $Y$ and still obtain a proper Young wall.

(b) A proper Young wall is said to be reduced if none of its columns contain a removable $\delta$.

Example 6.6. The first Young wall drawn below is reduced, but the second one is not reduced.
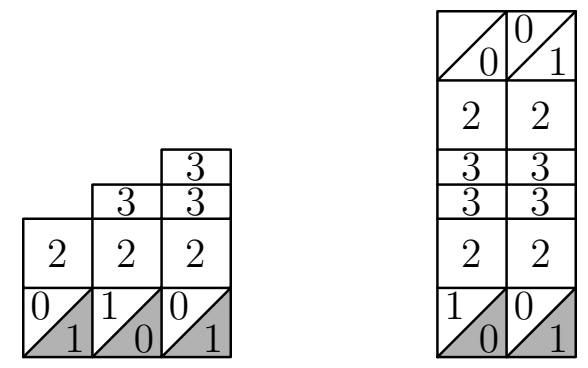

Let $\mathbf{Y}(\lambda)$ be the set of all reduced proper Young walls built on the groundstate wall $Y_{\lambda}$. We first prove that the set $\mathbf{Y}(\lambda)$ is stable under the Kashiwara operators.

Theorem 6.7. For all $i \in I$ and $Y \in \mathbf{Y}(\lambda)$, we have

$$
\tilde{e}_{i} Y \in \mathbf{Y}(\lambda) \cup\{0\} \quad \text { and } \quad \tilde{f}_{i} Y \in \mathbf{Y}(\lambda) \cup\{0\} .
$$

Hence the set $\mathbf{Y}(\lambda)$ has an affine crystal structure for the quantum affine algebra $U_{q}(\mathfrak{g})$. 
Proof. Let $Y \in \mathbf{Y}(\lambda)$ and fix an index $i \in I$. If $\tilde{e}_{i} Y \neq 0$, by definition of removable blocks, $\tilde{e}_{i} Y$ is a proper Young wall. Suppose that $\tilde{e}_{i} Y$ is not reduced. This means that removing an $i$-block for $Y$ has created a removable $\delta$ in $\tilde{e}_{i} Y$. Hence $Y$ must have a column of the form:

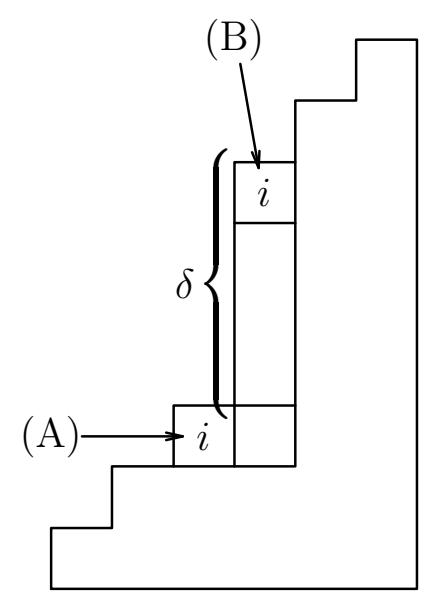

Here, the $i$-block at $(\mathrm{A})$ is removed to give $\tilde{e}_{i} Y$. Then, in this case, the rules for the action of Kashiwara operators tell us that the operator $\tilde{e}_{i}$ would remove the $i$-block at (B), not the one at (A), which is a contradiction. Hence $\tilde{e}_{i} Y$ is reduced.

Similarly, if $\tilde{f}_{i} Y \neq 0$, one can show that $\tilde{f}_{i} Y$ is a reduced proper Young wall.

\section{CRystal graphs For BASiC REPRESEntations}

In this section, we will prove that the crystal graph $B(\lambda)$ for the basic representation $V(\lambda)$ of the quantum affine algebra $U_{q}(\mathfrak{g})$ is isomorphic to the affine crystal $\mathbf{Y}(\lambda)$ consisting of all reduced proper Young walls built on the groundstate wall $Y_{\lambda}$. Hence the crystal $\mathbf{Y}(\lambda)$ would be the connected component of the crystal $\mathbf{F}(\lambda)$ contaning the ground-state wall $Y_{\lambda}$.

Theorem 7.1. There exists an isomorphism of $U_{q}(\mathfrak{g})$-crystals

$$
\mathbf{Y}(\lambda) \stackrel{\sim}{\longrightarrow} B(\lambda) \text { given by } Y_{\lambda} \longmapsto u_{\lambda},
$$

where $u_{\lambda}$ is the highest weight vector in $B(\lambda)$.

Proof. The rest of this section is devoted to proving this theorem. It suffices to show $\mathbf{Y}(\lambda) \cong \mathbf{P}(\lambda)$ as $U_{q}^{\prime}(\mathfrak{g})$-crystals. Let us define a map $\Psi: \mathbf{Y}(\lambda) \longrightarrow \mathbf{P}(\lambda)$ as follows: We read the top parts of each column in a reduced proper Young wall and write down a sequence of elements from $\mathbf{B}$, the perfect crystal of level 
1 , to obtain a $\lambda$-path. The rules for obtaining the paths are given below for each affine type in question. 
(a) $A_{n}^{(1)}(n \geq 1)$
$j-1 \longmapsto j$
$(1 \leq j \leq n)$,
$n \longmapsto 0$

(b) $A_{2 n-1}^{(2)}(n \geq 3)$

$\triangle$ or $0 \longmapsto 1$

$0 / 1$ or $1 / 0 \longmapsto 2$

\begin{tabular}{|l|l|}
$n$ & $\longmapsto$ \\
\hline$n-1$ & \\
&
\end{tabular}

\begin{tabular}{|l|l|}
\hline 2 & or 2 \\
\hline 1 & $1 / 0$ \\
\hline 1
\end{tabular}

\begin{tabular}{|c|}
\hline$j$ \\
\hdashline$j+1$
\end{tabular}

$\stackrel{j-1}{j-2} \longmapsto j \quad(4 \leq j \leq n)$

17 or $\triangle 1 \longmapsto \overline{1}$

(c) $D_{n}^{(1)}(n \geq 4)$

$\triangle$ or $0 \longmapsto 1$

$\not n$ or $n \longmapsto \bar{n}$

21 or $1 / 0 \longmapsto 2$

$\stackrel{n-1}{n-1}$ or $\begin{aligned} & n \Rightarrow \\ & n-1\end{aligned} \longmapsto n$

\begin{tabular}{|l|l|}
\hline 2 & or 2 \\
\hline 2 & $1 / 0$
\end{tabular}

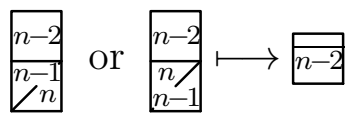

\begin{tabular}{|l|l}
$j-1$ \\
\hdashline$j-2$
\end{tabular}$\quad(4 \leq j \leq n-1)$

\begin{tabular}{|c|}
$j$ \\
\hline$j+1$
\end{tabular} $\bar{j} \quad(2 \leq j \leq n-3)$

$n-1$ or $\stackrel{n-1}{n} \longmapsto n$

17 or $\triangle 1 \longmapsto \overline{1}$ 
(d) $A_{2 n}^{(2)}(n \geq 2)$

$$
\begin{aligned}
& \square \longmapsto \emptyset \\
& \hline 0 \\
& \hline 0 \\
& \hline 1 \\
& \hline 0 \\
& \hline 0 \\
& 0
\end{aligned}
$$

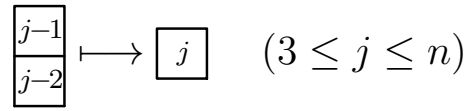

$$
\begin{aligned}
& \stackrel{n=1}{n n} \longmapsto \bar{n} \\
& \begin{array}{|c|c|c|}
\hline j \\
\hdashline j+1
\end{array} \quad(1 \leq j \leq n-1)
\end{aligned}
$$

(e) $D_{n+1}^{(2)}(n \geq 2)$

$$
\begin{aligned}
& \square \longmapsto \emptyset \\
& 0 \begin{array}{l}
0 \\
\hline 0
\end{array} 1 \\
& \begin{array}{ll}
1 \\
\hline 0 \\
0
\end{array}
\end{aligned}
$$$$
\square \longmapsto 0
$$$$
\begin{array}{|l|}
\hline n \\
\hline n
\end{array}
$$$$
\begin{gathered}
n-1 \\
\hdashline n \\
n
\end{gathered}
$$

$\stackrel{j-1}{j-2} \longmapsto \quad j \quad(3 \leq j \leq n)$

\begin{tabular}{|c|c|c|}
\hline$j$ & & \\
\hdashline$j+1$ & & $(1 \leq j \leq n-2)$
\end{tabular}

(f) $B_{n}^{(1)}(n \geq 3)$

$\square$ or $\square \longmapsto 1$

011 or $1 / 0 \longmapsto 2$

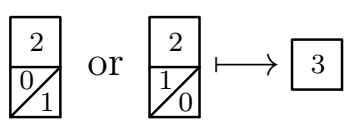

$$
\stackrel{n}{n} \longmapsto \bar{n}
$$$$
\begin{array}{|c|c|}
\hline n-1 \\
\hdashline n \\
\hline n
\end{array}
$$

$\stackrel{j-1}{j-2} \longmapsto \quad(4 \leq j \leq n)$

\begin{tabular}{|c|c|c|}
\hline$j$ \\
\end{tabular}

$1 /$ or $\triangle 1$

$$
n \longmapsto 0
$$


It is clear that this map sends the ground-state walls to the appropriate ground-state paths and that the image does indeed lie in the set $\mathbf{P}(\lambda)$ of $\lambda$ paths. Moreover, the surjectivity of $\Psi$ follows immediately from the definition, and the injectivity of $\Psi$ follows from the fact that the proper Young walls in $\mathbf{Y}(\lambda)$ are reduced. Hence we have only to show that the map $\Psi$ commutes with Kashiwara operators. We give a proof of our claim only for the case of $B_{n}^{(1)}$. Other cases may be proved in similar manners and are less complicated.

Let $Y=\left(y_{k}\right)_{k=0}^{\infty} \in \mathbf{Y}(\lambda)$, where $y_{k}$ denotes the $k$-th column of $Y$ (counting from the right to the left) and let $\mathbf{p}=(\mathbf{p}(k))_{k=0}^{\infty}=\Psi(Y)$ be the image of $Y$ under $\Psi$. Recall that the action of the Kashiwara operators is determined by the $i$-signatures of $Y$ and $\mathbf{p}$. Fix an index $i \in I$ and consider the Kashiwara operator $\tilde{f}_{i}$.

(1) $i=0$ : Suppose that the top of the $k$-th column $y_{k}$ of $Y$ is the cube 1 . The column $y_{k}$ is certainly not 0 -removable. If it is 0 -admissible, we would assign $\mathrm{a}+$ to the column, which is what we would also do with the corresponding element $\mathbf{p}(k)=\overline{1}$. If it is not 0 -admissible, then the top of the column $y_{k-1}$ must be either $\angle 0$ or $1 / 0$. In each of the two cases, $y_{k-1}$ is neither 0 -admissible nor 0 -removable, so we would assign nothing to $y_{k}$ and $y_{k-1}$.

Now, consider the corresponding terms $\mathbf{p}(k)$ and $\mathbf{p}(k-1)$ of the path $\mathbf{p}=$ $(\mathbf{p}(k))_{k=0}^{\infty}=\Psi(Y)$. We have $\mathbf{p}(k)=1$ and $\mathbf{p}(k-1)=1$ or 2 . We assign + for $\mathbf{p}(k)=\overline{1}$ and - for $\mathbf{p}(k-1)=1$ or 2 , which cancel out to give nothing. Thus the resulting 0 -signatures coincide with each other.

Similarly, we can verify that we would assign the same 0-signatures to $y_{k}$ and $\mathbf{p}(k)$ for the other cases of $y_{k}$. In Table 1, we list all the possible nontrivial cases for $i=0$.

(2) $i=1$ : This case is quite similar to the $i=0$ case.

(3) $i=2$ : We list all the possible nontrivial cases in Table 2 .

(4) $3 \leq i \leq n-2$ : We list all the possible nontrivial cases in Table 3 .

(5) $i=n-1$ : We list all the possible nontrivial cases in Table 4 .

(6) $i=n$ : Observe that the top parts of the columns of $Y$ that have a nontrivial contribution to the $n$-signature of $Y$ have the following form.

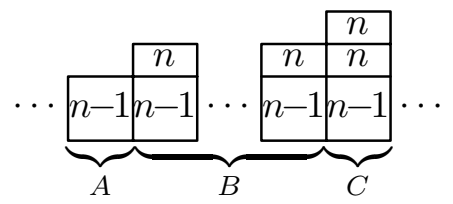


Here, we do not exclude the possibility of some of the parts A, B, or C missing in this drawing. The ground-state wall of weight $\Lambda_{n}$ could also be considered as a degenerate case of the above. It suffices to verify that the $n$-signature for this segment and that of the corresponding part in the path $\mathbf{p}$ are the same.

In the table given below, we list all the possible combinations for the parts $\mathrm{A}, \mathrm{B}$ and $\mathrm{C}$, and write down their $n$-signatures.

\begin{tabular}{|c|c|c|c|c|c|c|}
\hline \multicolumn{3}{|c|}{ presence of } & \multicolumn{3}{|c|}{ signature } & total \\
\hline A & B & $\mathrm{C}$ & A & B & $\mathrm{C}$ & signature \\
\hline yes & yes & yes & + & & - & \\
\hline yes & yes & no & + & + & & ++ \\
\hline yes & no & yes & + & & - & \\
\hline yes & no & no & ++ & & & ++ \\
\hline no & yes & yes & & - & - & -- \\
\hline no & yes & no & & -+ & & -+ \\
\hline no & no & yes & & & -- & -- \\
\hline
\end{tabular}

Now, one can easily verify that the $n$-signature for the above segment of $Y$ coincides with that of the corresponding part in the path $\mathbf{p}$.

$$
\cdots \otimes \overbrace{\substack{n \\++} \otimes \overbrace{-+}^{0} \otimes \cdots \otimes \underbrace{}_{-+} 0}^{A} \otimes \overbrace{\underbrace{\bar{n}}_{--}}^{C} \otimes \cdots
$$

Thus, we have shown that for all indices $i \in I$, the $i$-signature of the reduced proper Young wall $Y$ is identical to that of the path $\mathbf{p}=\Psi(Y)$. It is now straightforward to verify that the action of Kashiwara operators is compatible with the identification of $Y$ with $\mathbf{p}$. Here are some examples.

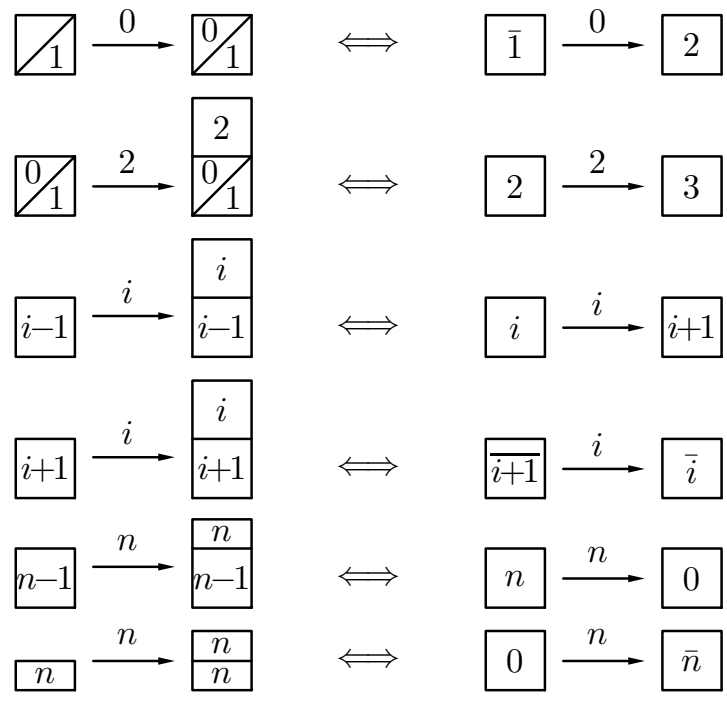

Therefore, we obtain the desired crystal isomorphism $\Psi: \mathbf{Y}(\lambda) \stackrel{\sim}{\longrightarrow} \mathbf{P}(\lambda)$. 


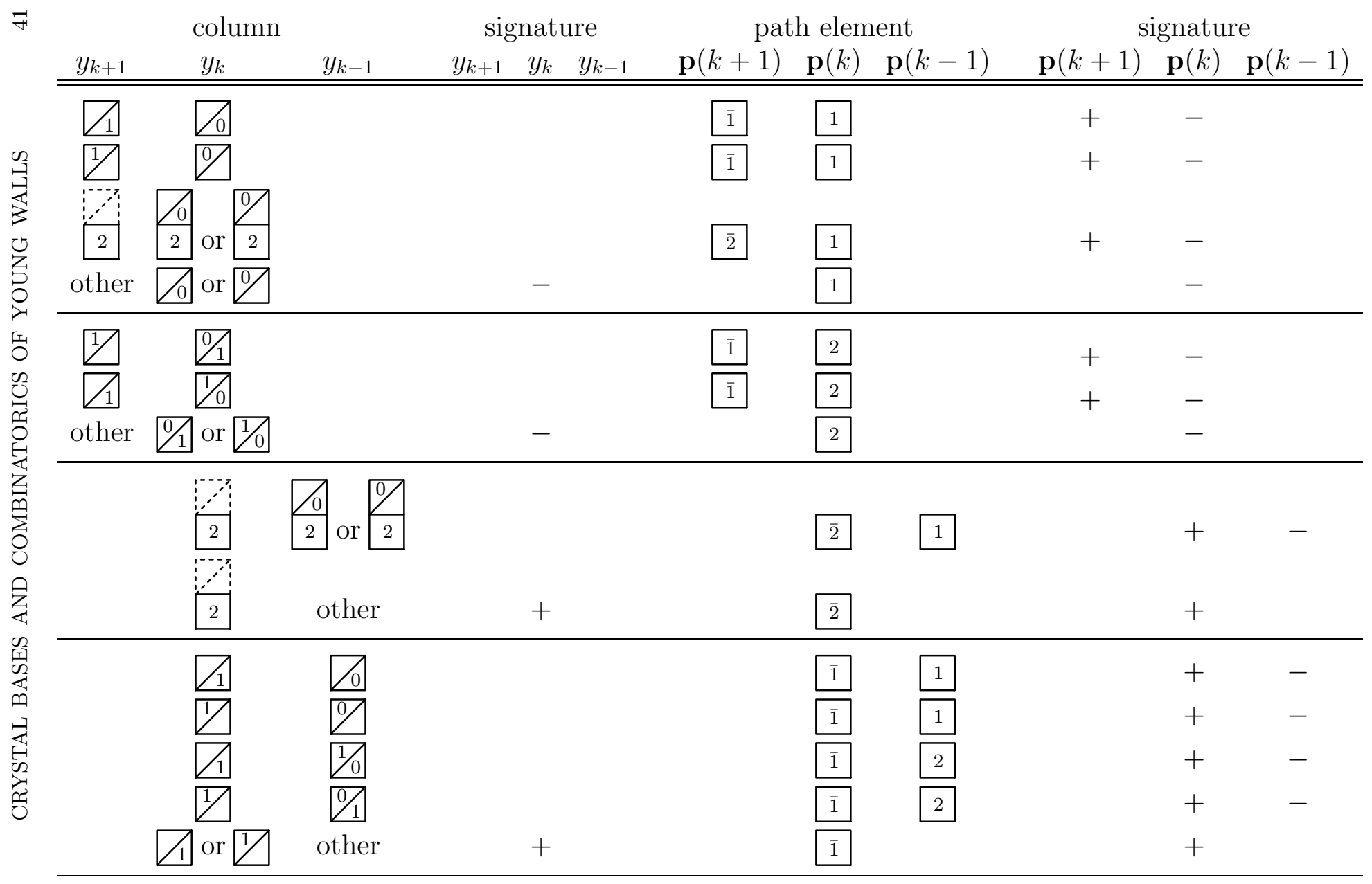

TABLE $1 . i=0$ 


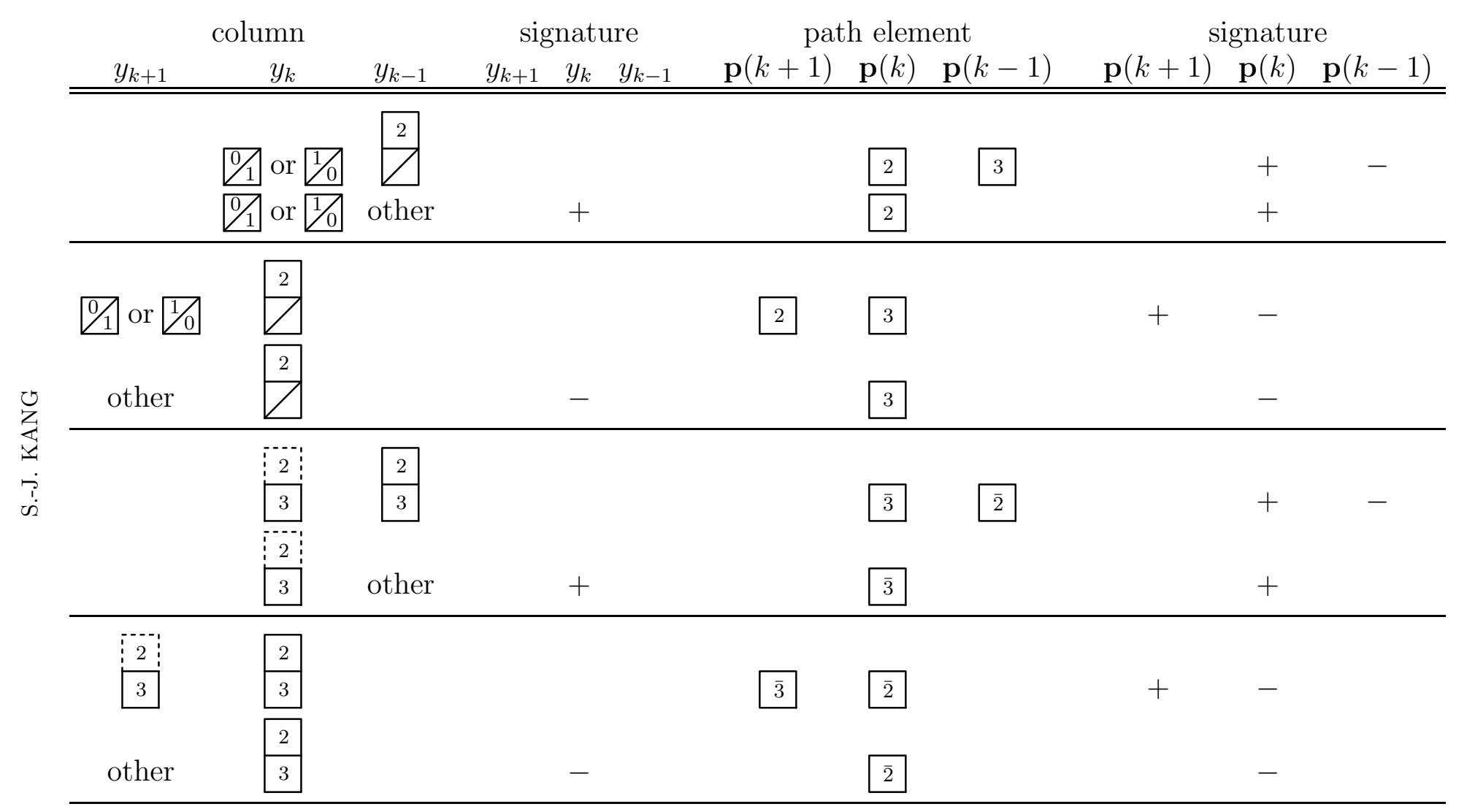

TABle $2 . i=2$ 


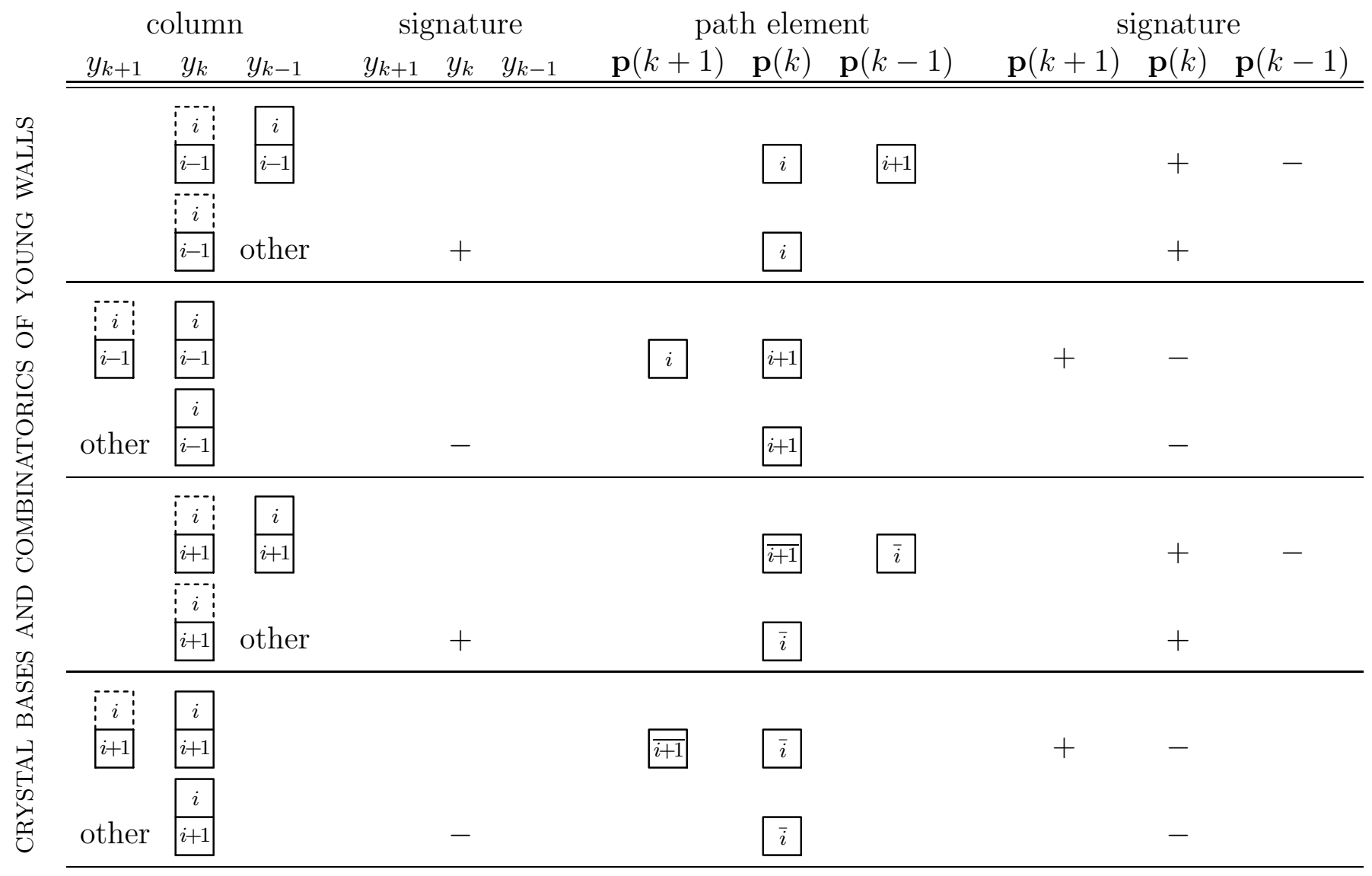

TABLE $3.3 \leq i \leq n-2$ 


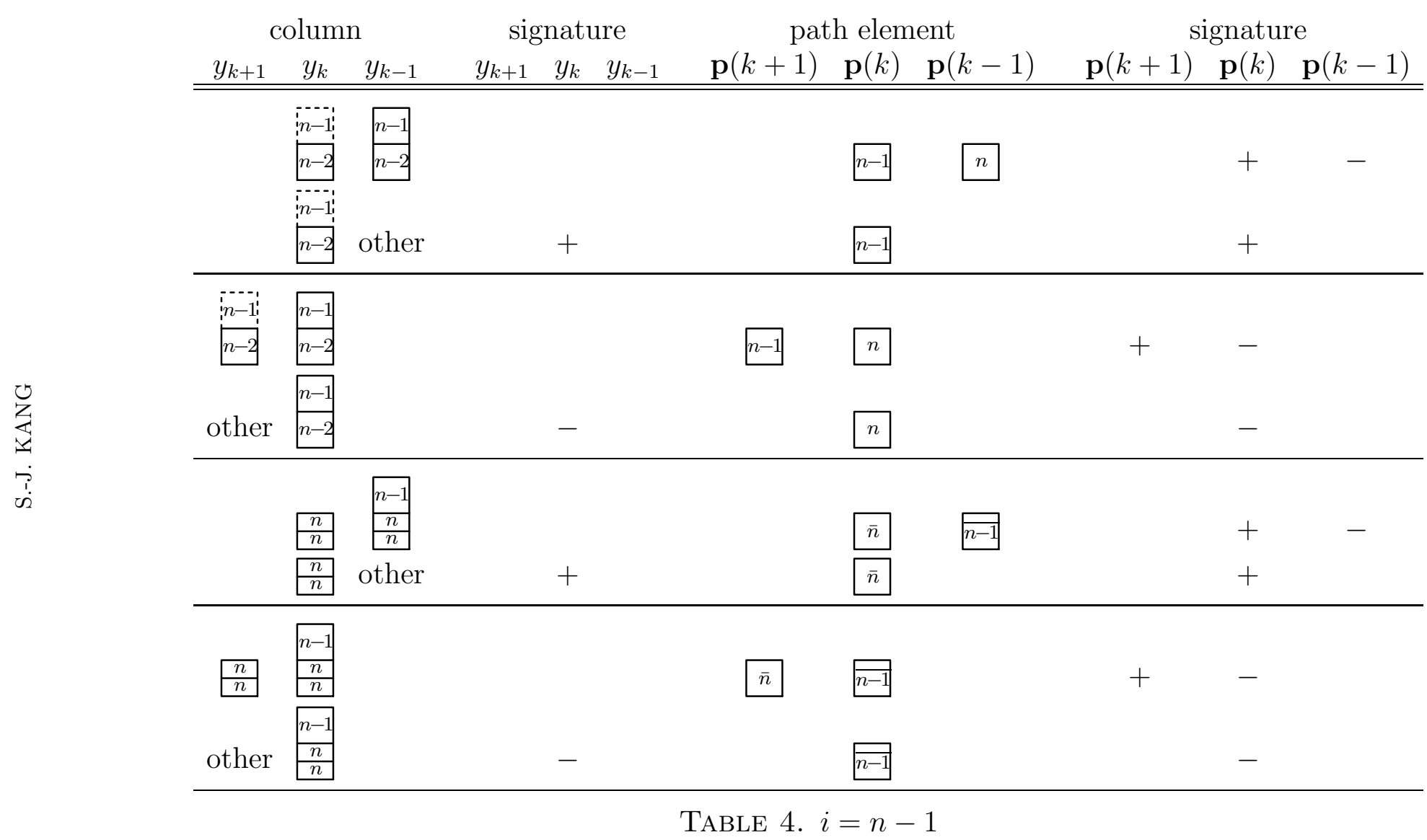


Example 7.2. In this example, we illustrate the top parts of the affine crystal $\mathbf{Y}(\lambda)$ consisting of reduced proper Young walls. Compare them with the path realization of crystal graphs given in Section 4.

(a) The crystal $\mathbf{Y}\left(\Lambda_{0}\right)$ for $A_{2}^{(1)}$

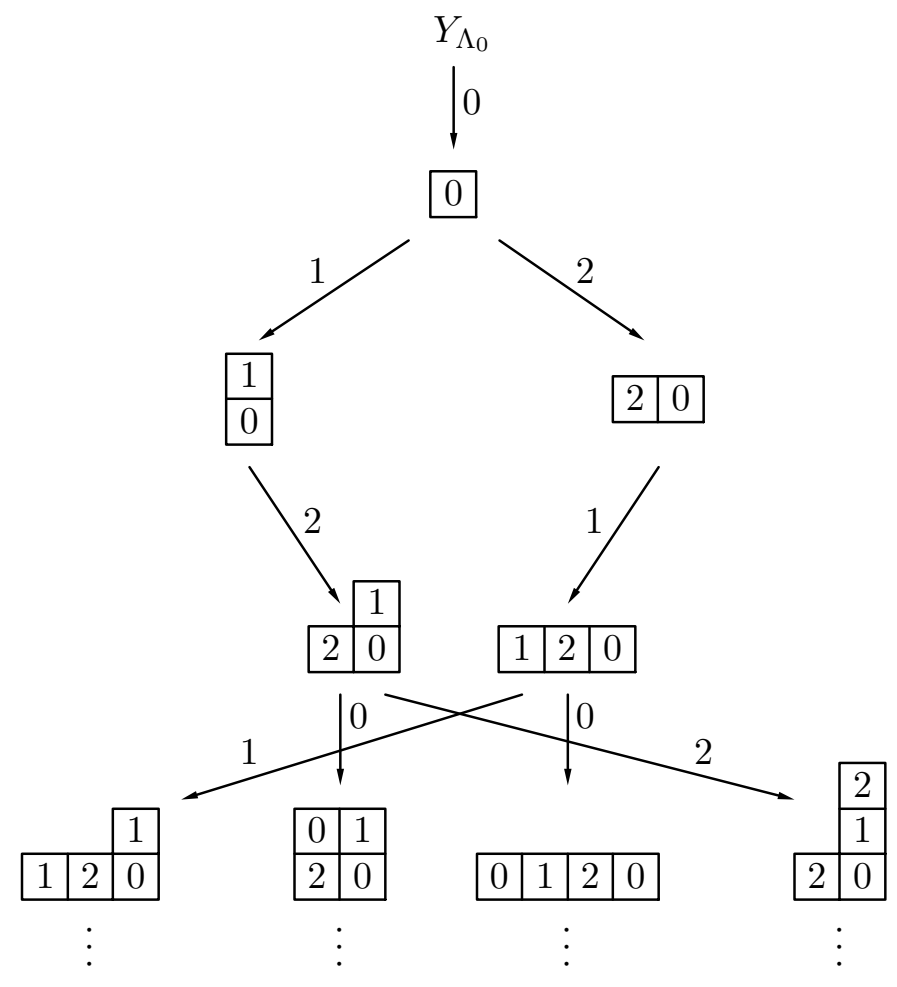


(b) The crystal $\mathbf{Y}\left(\Lambda_{0}\right)$ for $A_{5}^{(2)}$

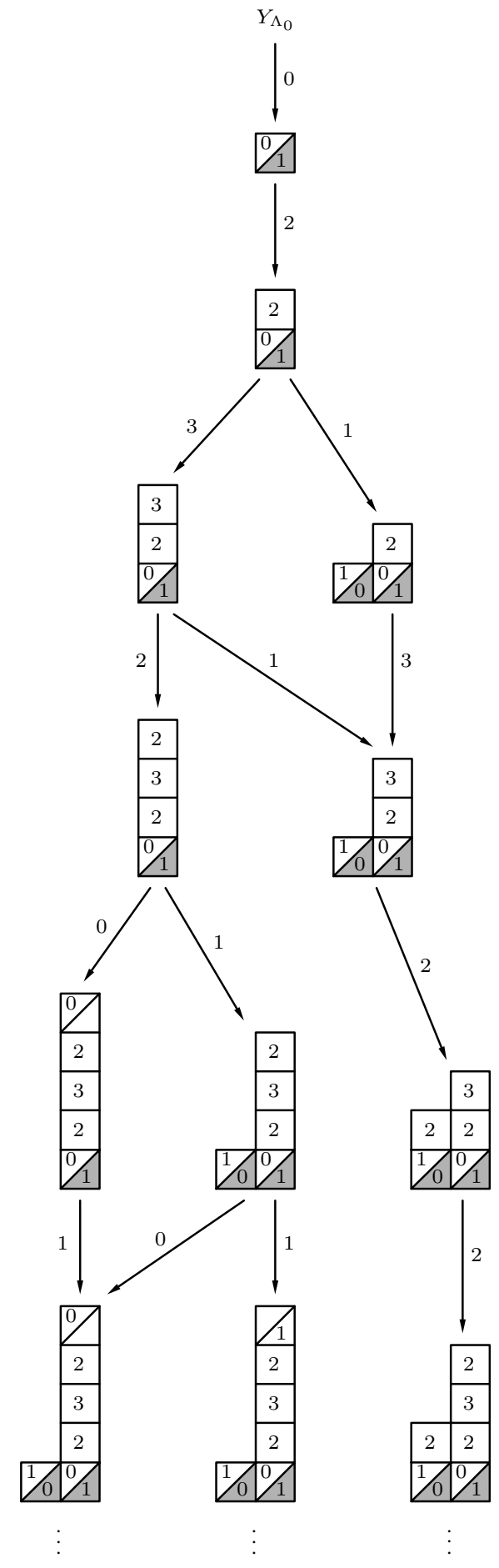


(c) The crystal $\mathbf{Y}\left(\Lambda_{0}\right)$ for $D_{4}^{(1)}$

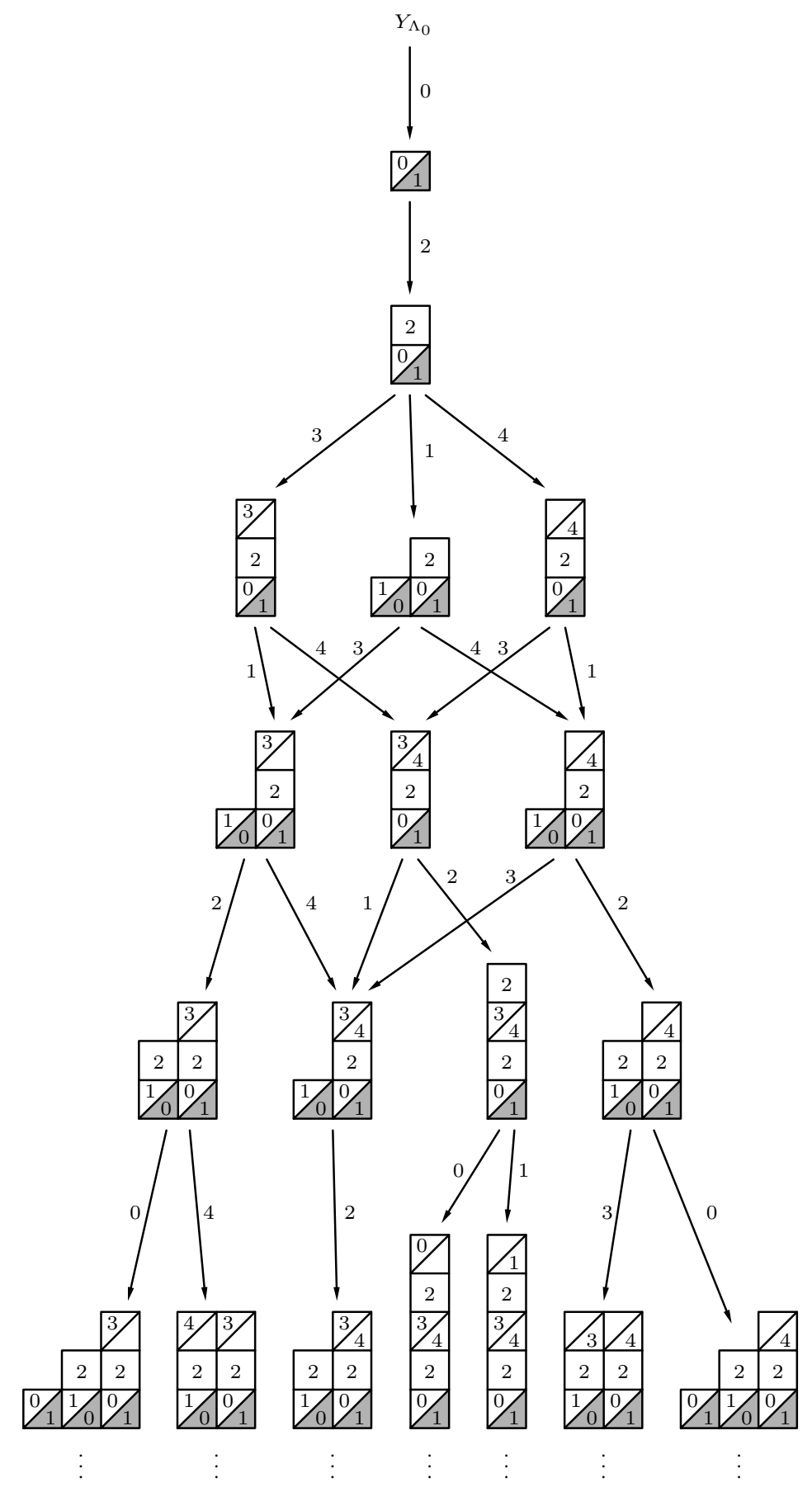


(d) The crystal $\mathbf{Y}\left(\Lambda_{0}\right)$ for $A_{4}^{(2)}$

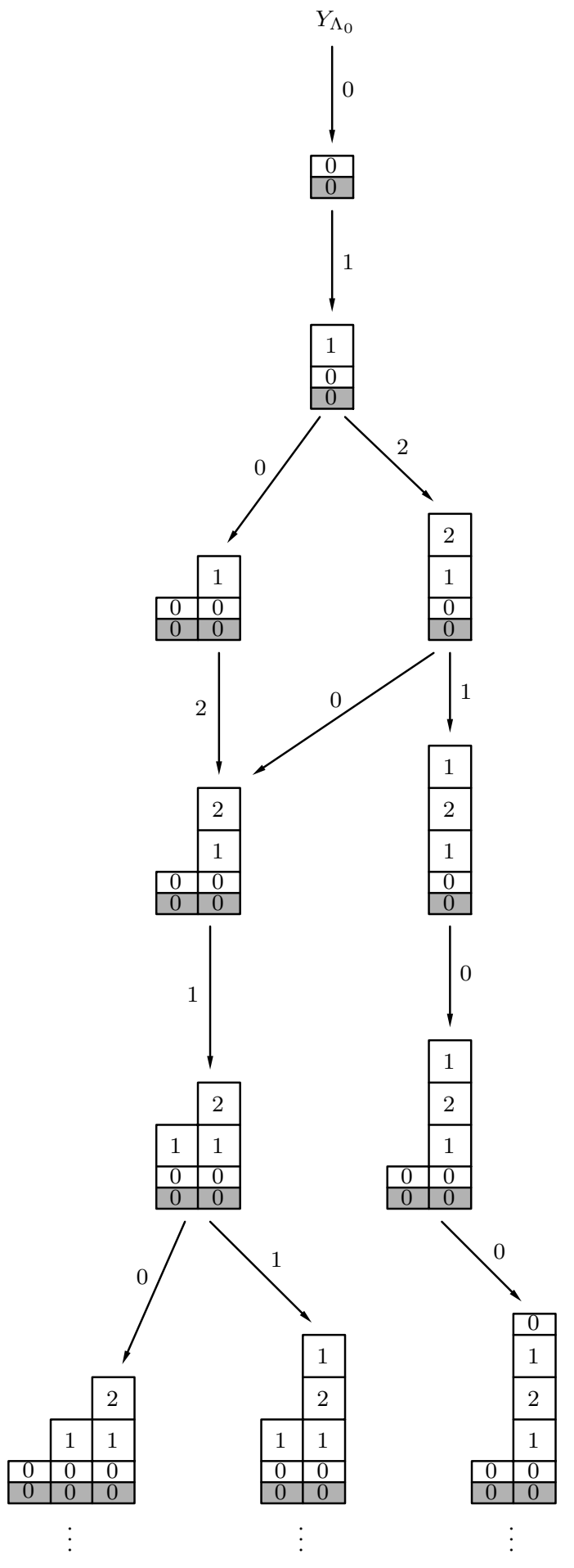


(e) The crystal $\mathbf{Y}\left(\Lambda_{0}\right)$ for $D_{3}^{(2)}$

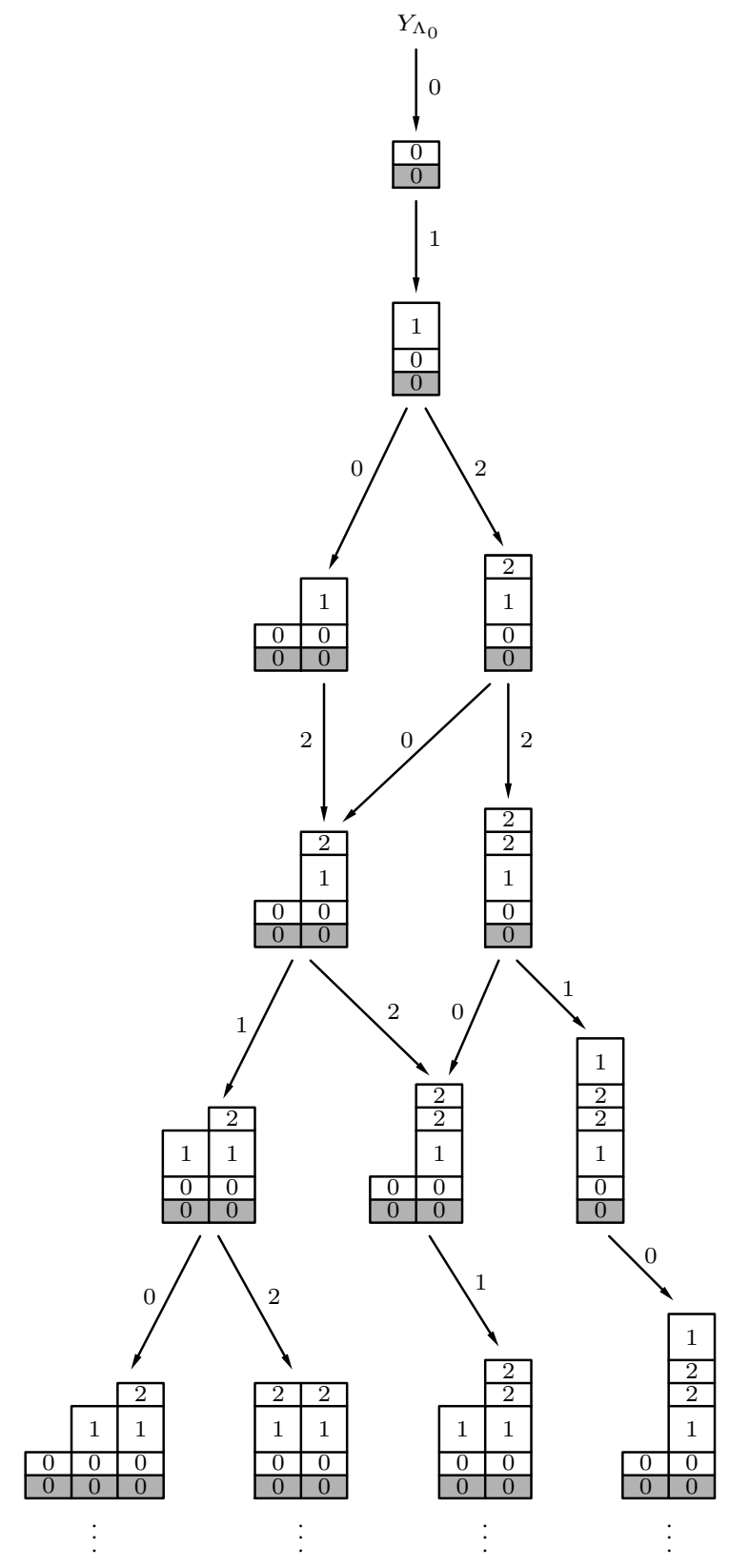


(f) The crystal $\mathbf{Y}\left(\Lambda_{0}\right)$ for $B_{3}^{(1)}$

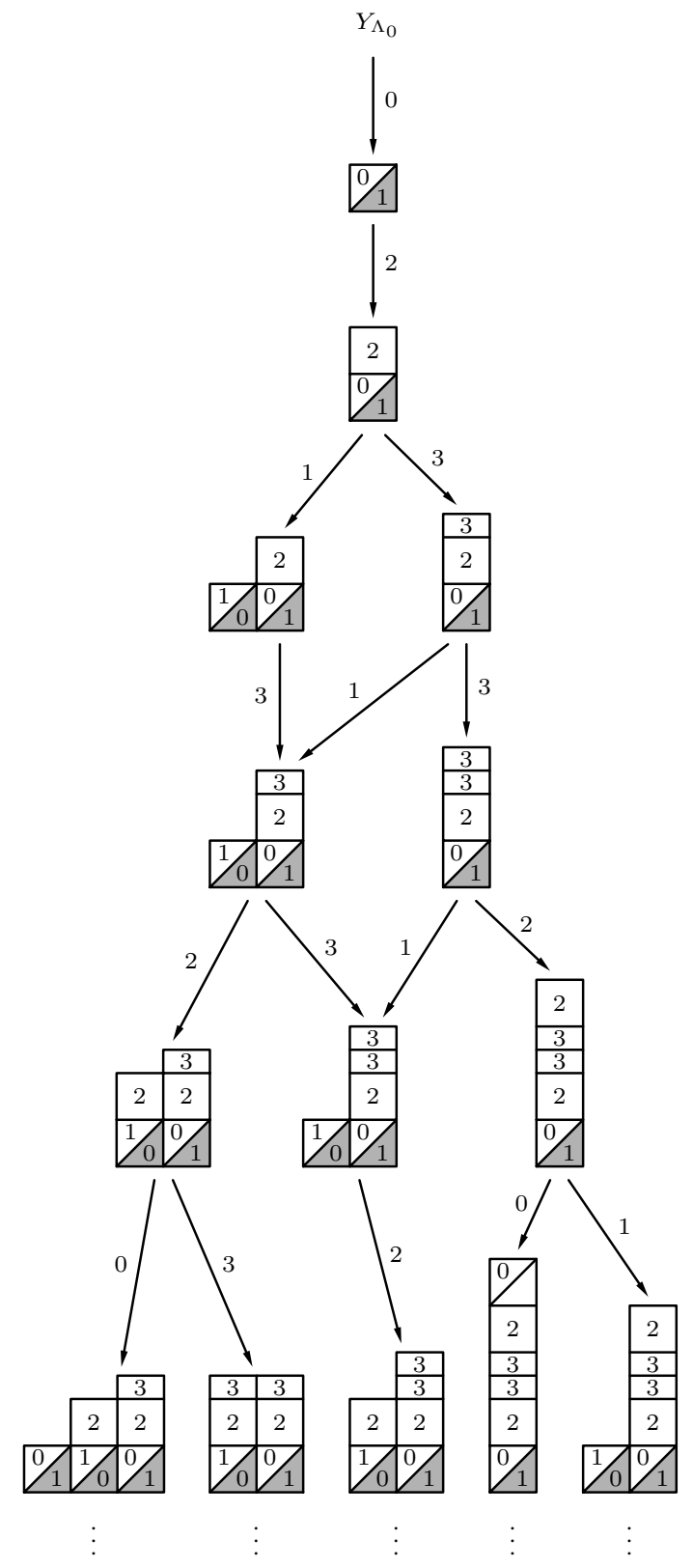


(g) The crystal $\mathbf{Y}\left(\Lambda_{3}\right)$ for $B_{3}^{(1)}$

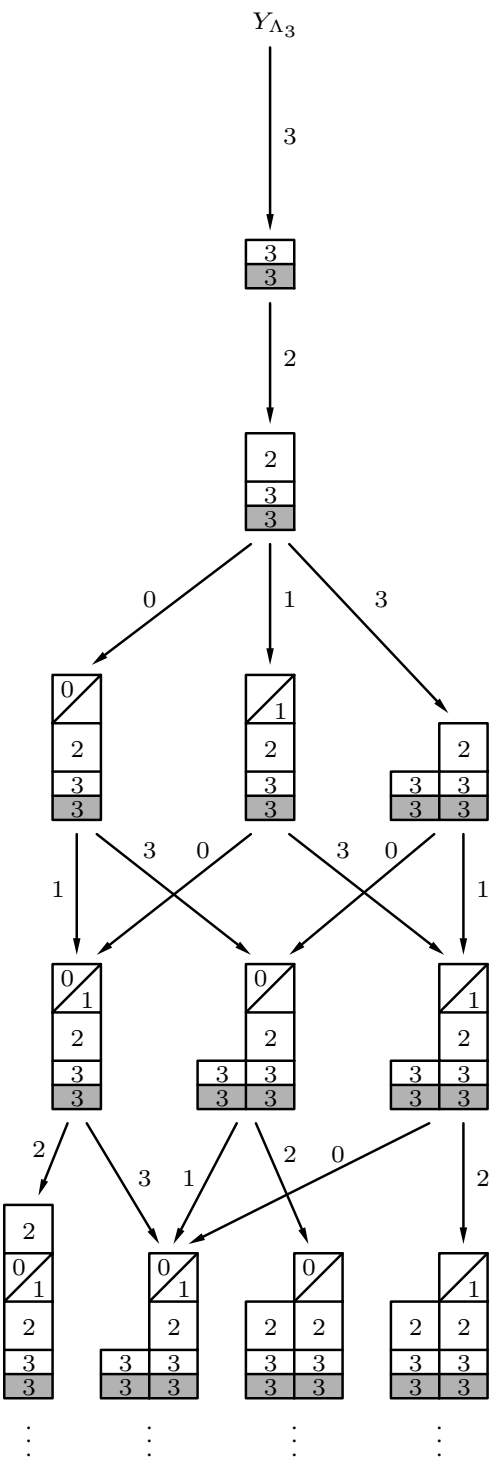




\section{REFERENCES}

[1] S. Ariki, On decomposition number of Hecke algebra of $G(m, 1, n)$, J. Math. Kyoto Univ. 36 (1996), 789-808.

[2] R. Dipper, G. James, Representations of Hecke algebras of the general linear group, Proc. Lond. Math. Soc. 52 (1986), 20-52.

[3] R. Dipper, G. James, Blocks and idempotents of the Hecke algebra of the general linear group, Proc. Lond. Math. Soc. 54 (1987), 57-82.

[4] F. Goodman, H. Wenzl, Crystal bases of quantum affine algebras and affine KazhdanLusztig polynomials, Internat. Math. Res. Notices 5 (1999), 251-275.

[5] J. Hong, S.-J. Kang, Crystal graphs for basic representations of the quantum affine algebra $U_{q}\left(C_{2}^{(1)}\right)$, RIM-GARC preprint 99-43 (1999), submitted.

[6] M. Jimbo, K. C. Misra, T. Miwa, and M. Okado, Combinatorics of representations of $U_{q}(\widehat{\mathfrak{s l}}(n))$ at $q=0$, Comm. Math. Phys. 136 (1991), 543-566.

[7] V. Kac, Infinite Dimensional Lie Algebras, Cambridge University Press, 3rd ed., 1990.

[8] S.-J. Kang, M. Kashiwara, K. C. Misra, T. Miwa, T. Nakashima, and A. Nakayashiki, Affine crystals and vertex models, Int. J. Mod. Phys. A. Suppl. 1A (1992), 449-484.

[9] _ Perfect crystals of quantum affine Lie algebras, Duke Math. J. 68 (1992), 499607.

[10] M. Kashiwara, Crystalizing the q-analogue of universal enveloping algebras, Comm. Math. Phys. 133 (1990), 249-260.

[11] _ On crystal bases of the q-analogue of universal enveloping algebras, Duke Math. J. 63 (1991), 465-516.

[12] _ Crystal bases and Littelmann's refined Demazure character formula, Duke Math. J. 71 (1993), 839-858.

[13] _ Crystal bases of modified quantized enveloping algebra, Duke Math. J. 73 (1994), 383-413.

[14] M. Kashiwara, T. Nakashima, Crystal graphs for representations of the q-analogue of classical Lie algebras, J. Algebra 165 (1994), 295-345.

[15] A. Lascoux, B. Leclerc, J-Y. Thibon, Hecek algebras at roots of unity and crystal bases of quantum affine algebras, Commun. Math. Phys. 181 (1996), 205-263.

[16] G. Lusztig, Canonical bases arising from quantized enveloping algebras, J. Amer. Math. Soc. 3 (1990), 447-498.

[17] K. Misra and T. Miwa, Crystal base for the basic representation of $U_{q}(\widehat{\mathfrak{s l}}(n))$, Comm. Math. Phys. 134 (1990), 79-88.

Department of Mathematics, Seoul National University, Seoul 151-742, KoREA

E-mail address: sjkang@math.snu.ac.kr 